\title{
Polyoxometalate-Based Metal-Organic Frameworks with Unique High-Nuclearity Water Clusters
}

Ze-Yu Du, ${ }^{\dagger}$ Yan-Zhao Yu, ${ }^{\dagger}$ Ya-Lin Hong, ${ }^{\dagger}$ Ning-Fang Li, ${ }^{\dagger}$ Ye-Min Han, ${ }^{\dagger}$ Jia-Peng Cao, ${ }^{\dagger}$ Qi Sun,,$\dot{+}$ Hua Mei ${ }^{*}+\grave{\dagger}$ and Yan $\mathrm{Xu}^{*}, \dot{\dagger}$

†College of Chemical Engineering, State Key Laboratory of Materials-Oriented Chemical Engineering, Nanjing Tech University, Nanjing 210009, P. R. China

\School of Chemistry and Chemical Engineering, Liaoning Normal University, Dalian 116029, China.

Corresponding authors: Hua Mei and Yan Xu

E-mails: meihua@njtech.edu.cn and yanxu@njtech.edu.cn 


\section{Content}

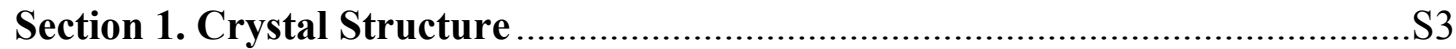

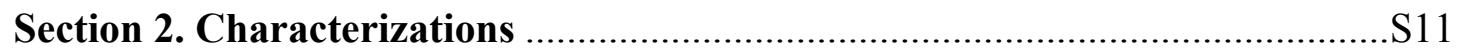

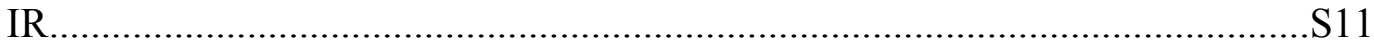

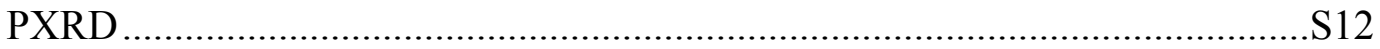

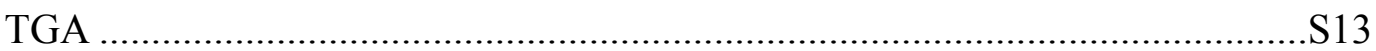

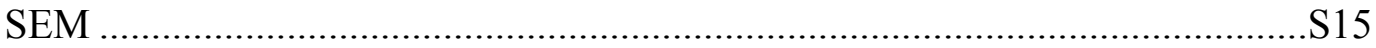

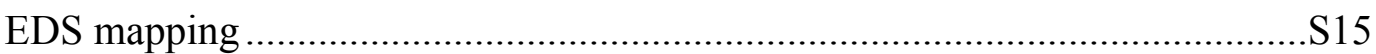

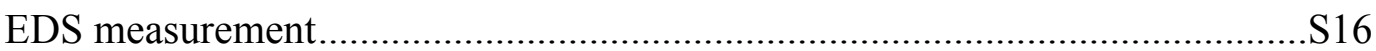

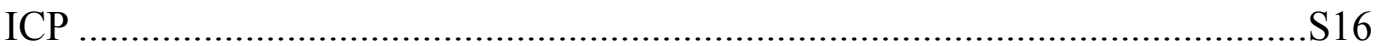

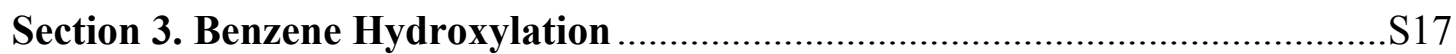

The Procedure of the Catalysis for the Oxidation of Benzene............................S17

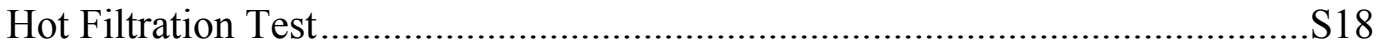

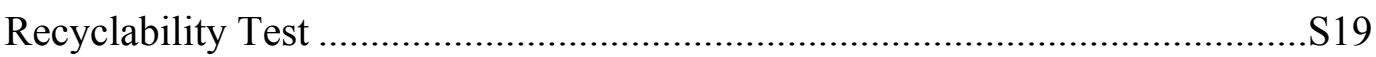

IR

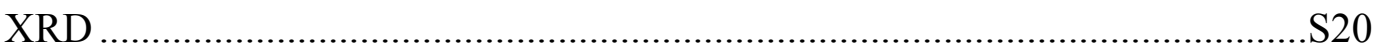

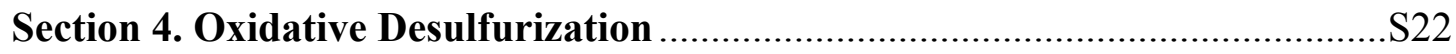

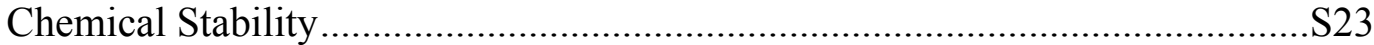

The Investigation of Optimal Reaction Condition...........................................S24

Stability Test for the Compound 2 as Catalyst ...............................................S25

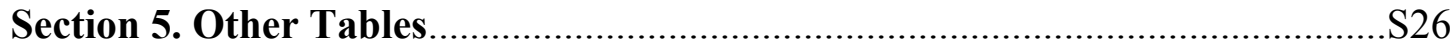

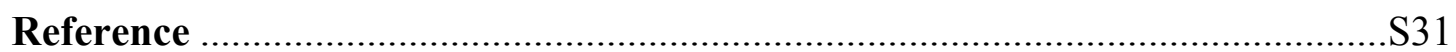




\section{Section 1. Crystal Structure}

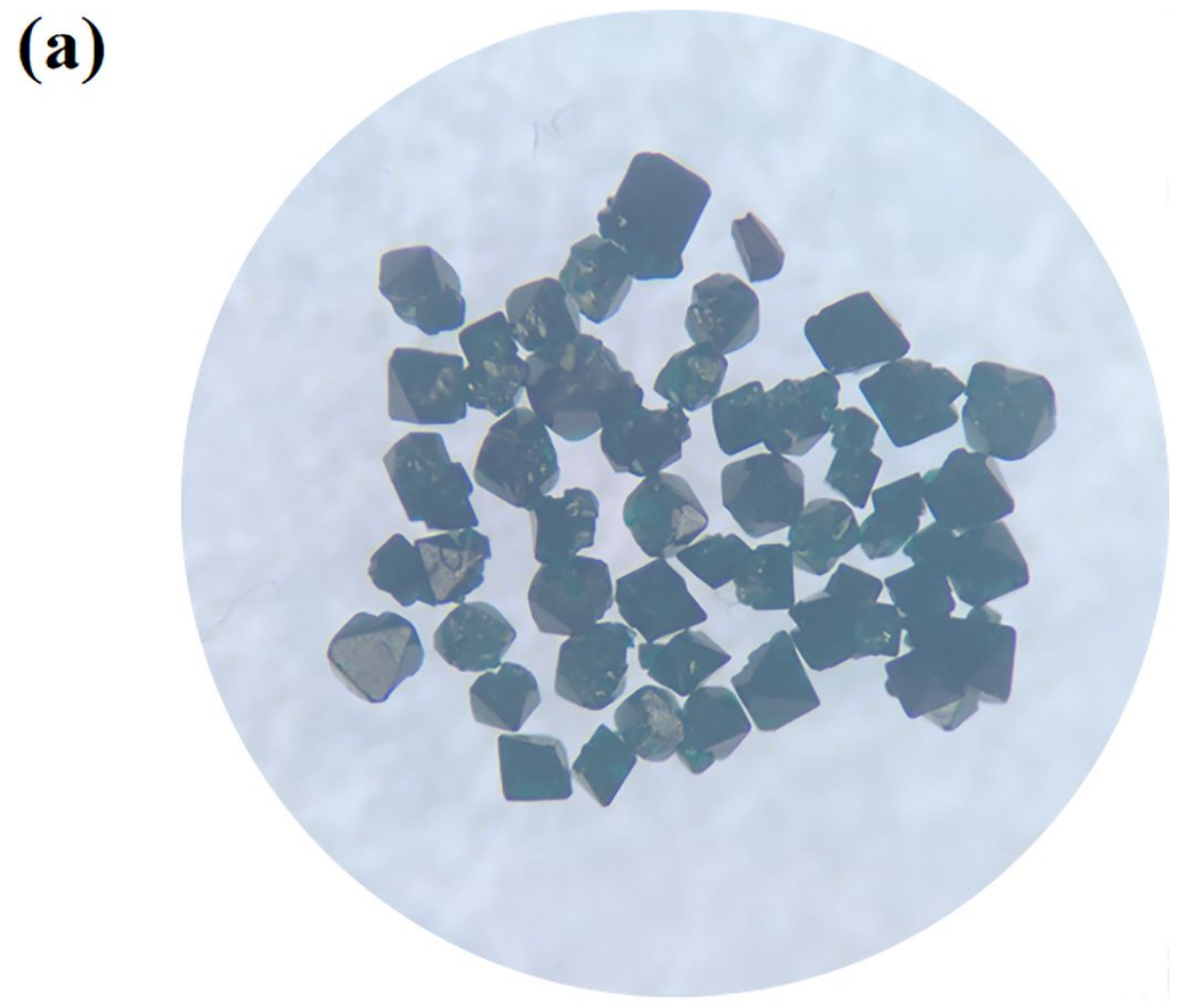

(b)

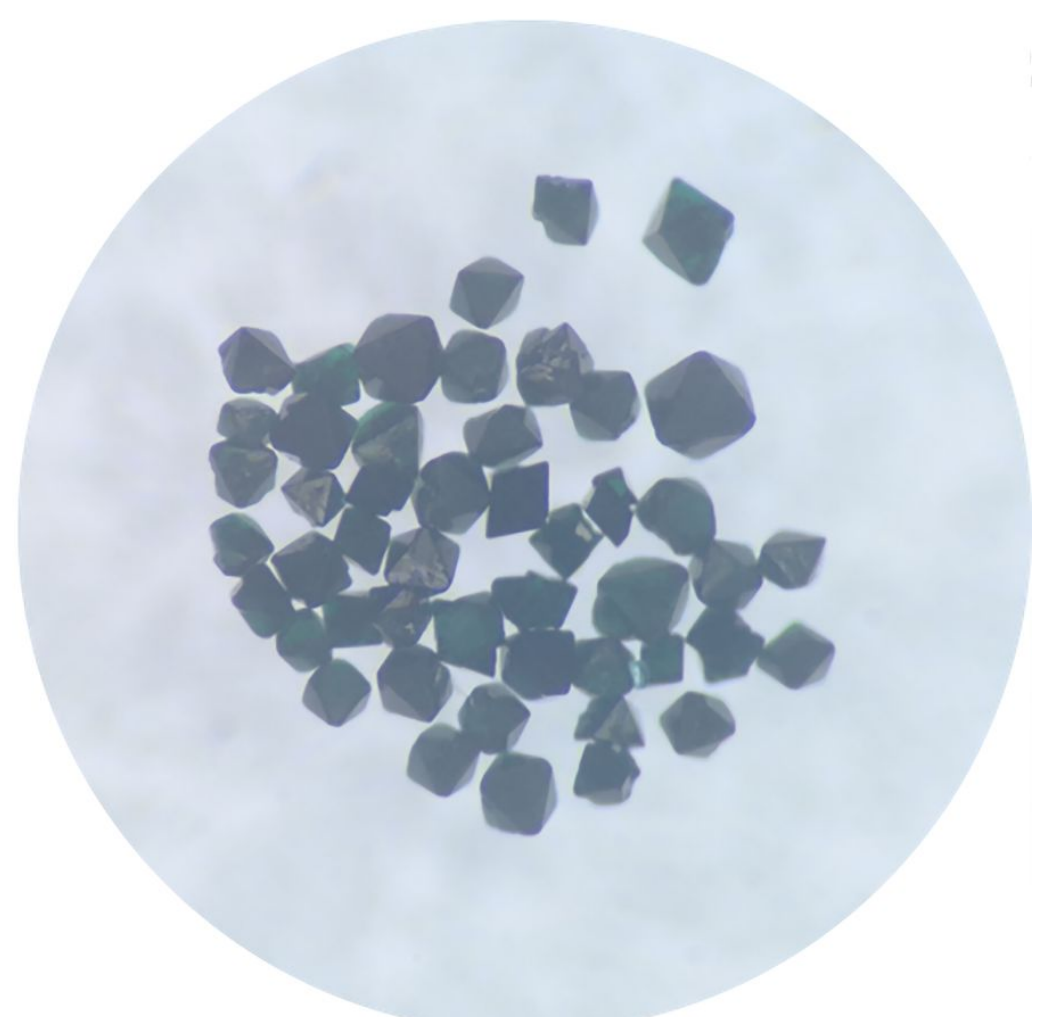

Figure S1. (a) The crystal image of compound 1 under optical microscope; (b) the crystal image of compound $\mathbf{2}$ under optical microscope. 
(a)

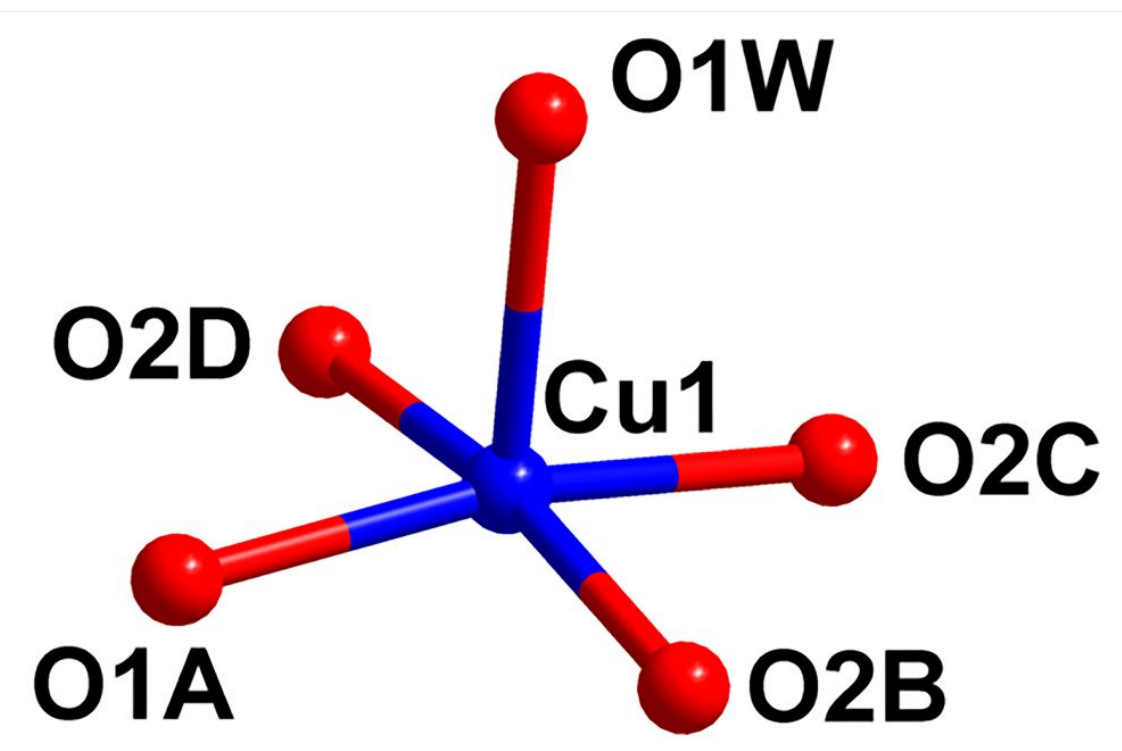

(b)

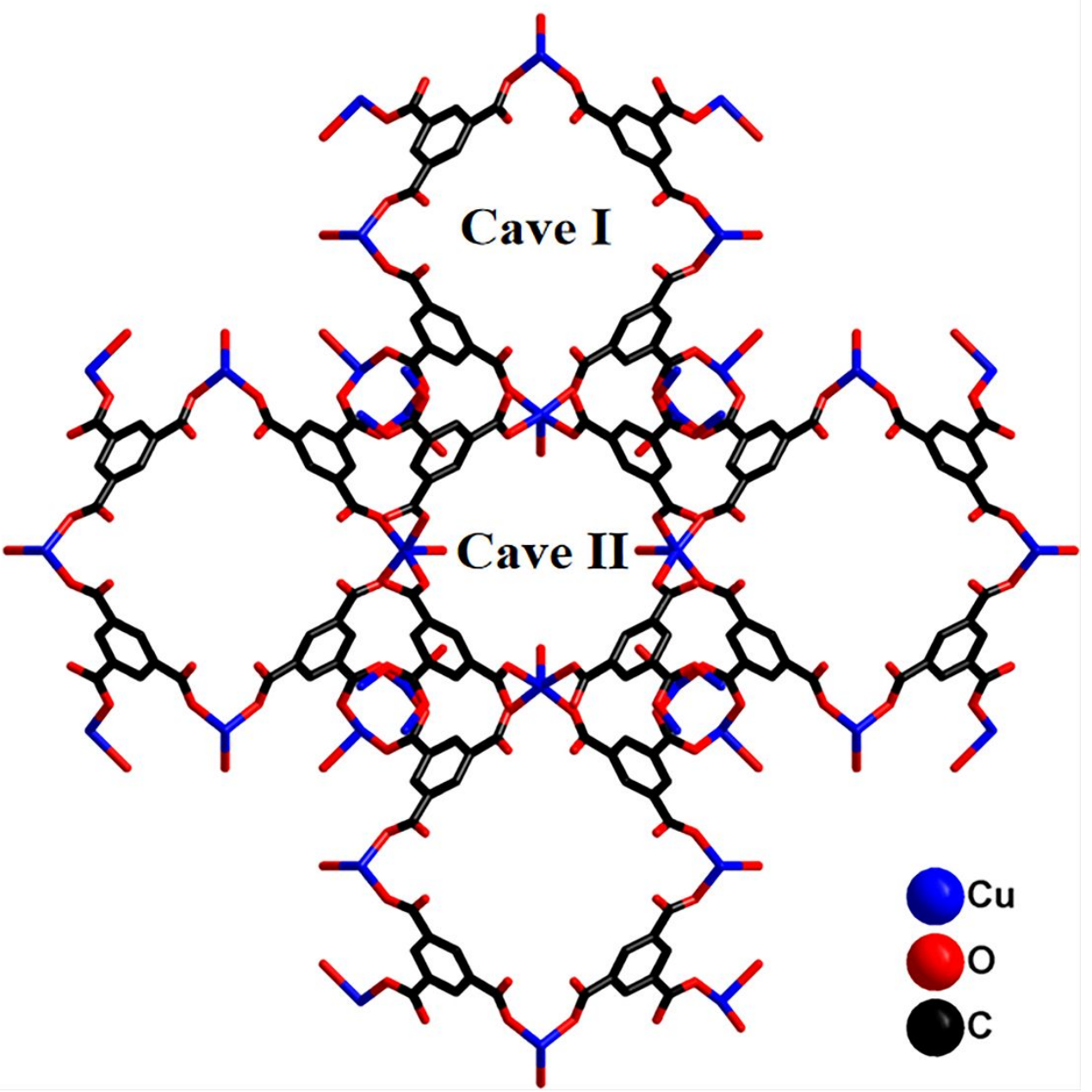

Figure S2. (a) The coordination environment of $\mathrm{Cu}$ 1; (b) the 2D structure of the metal organic framework $\mathrm{Cu}_{3}(\mathrm{BTC})_{2}$ view along $b$ axis. 
(a)

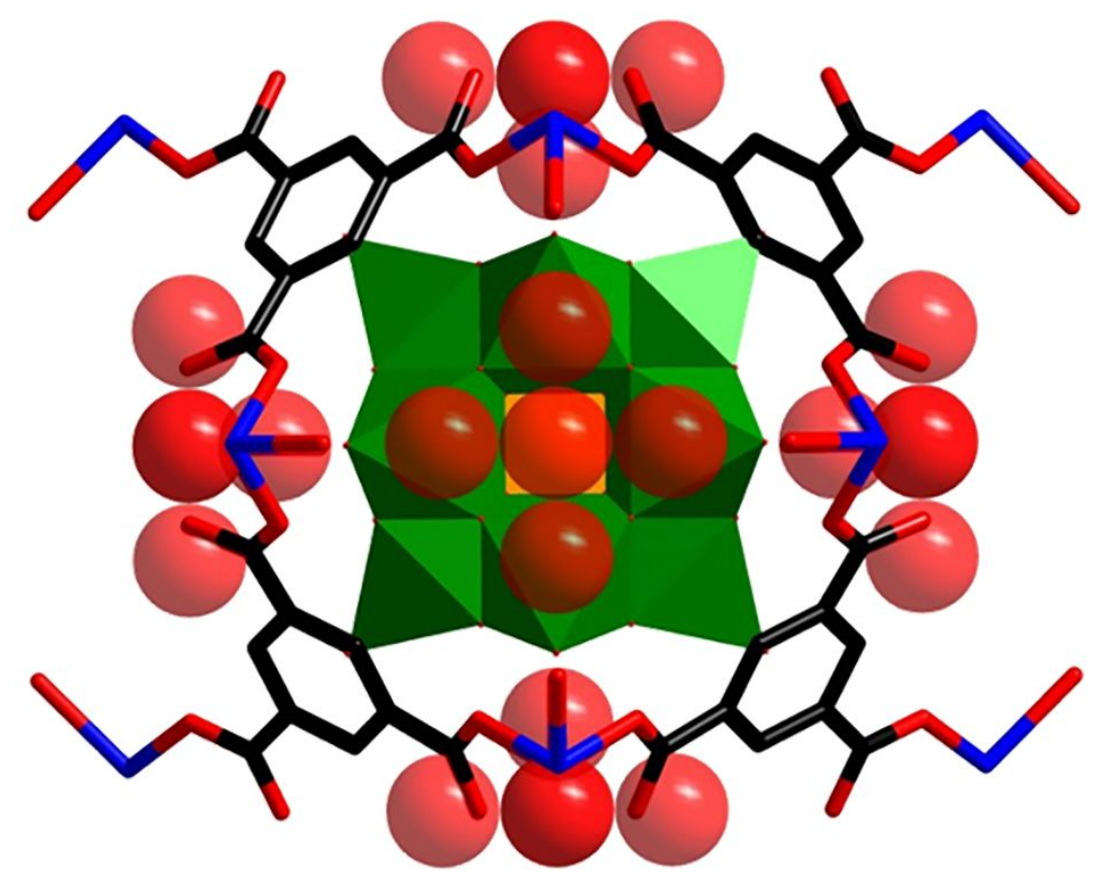

(b)

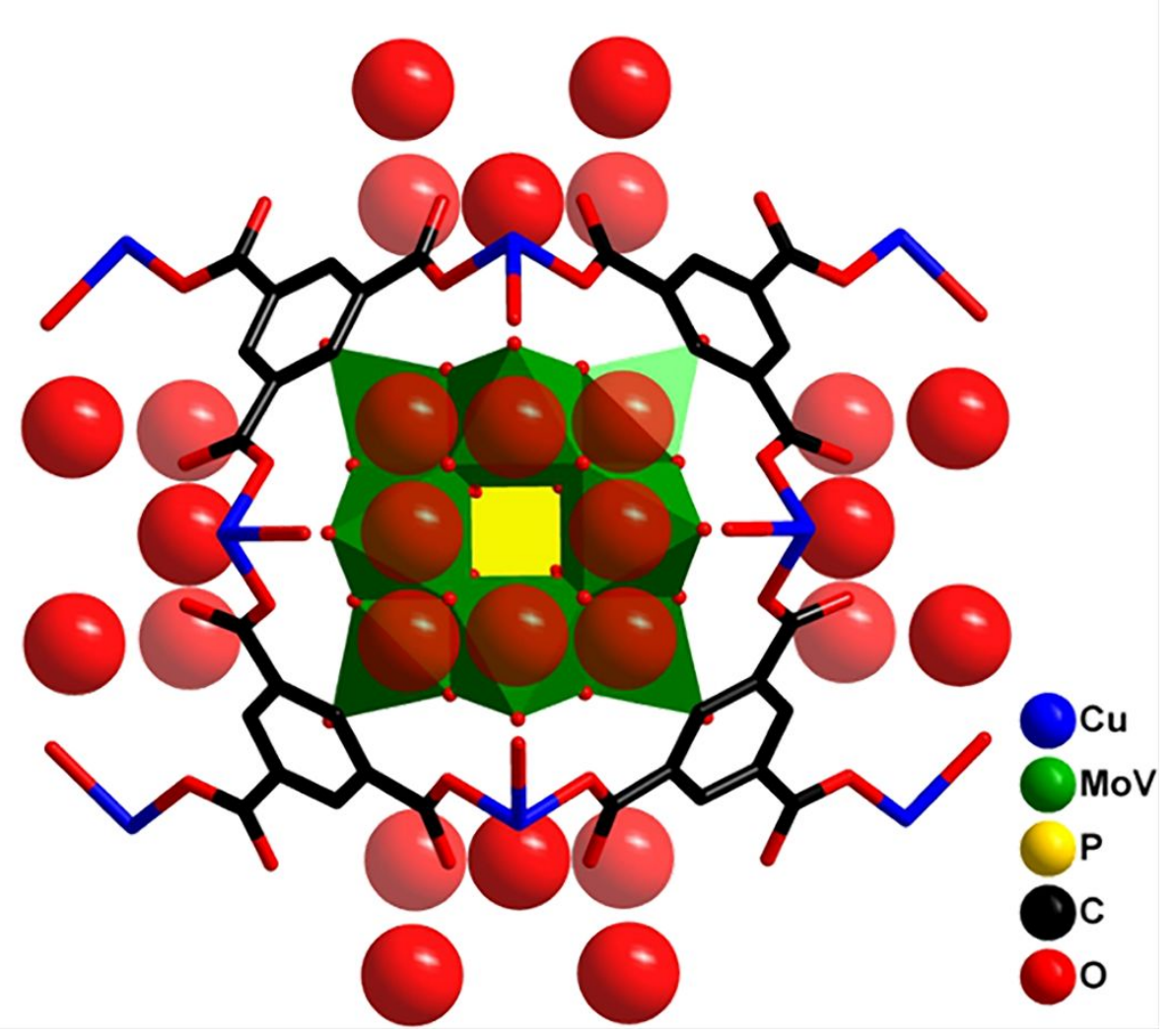

Figure S3. (a) Stick and polyhedral representation of compound 1; (b) stick and polyhedral representation of compound $\mathbf{2}$. 
(a)

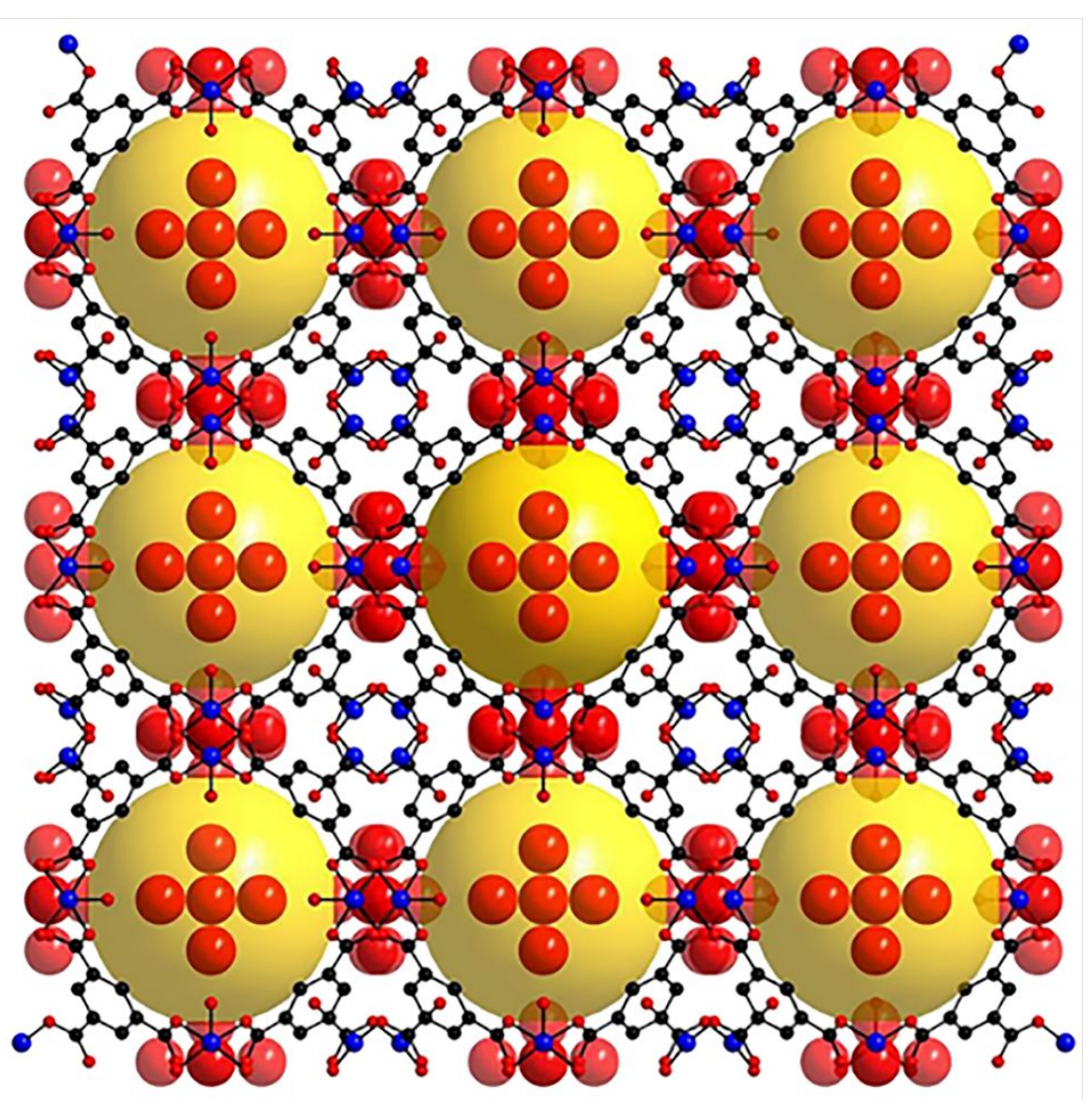

(b)

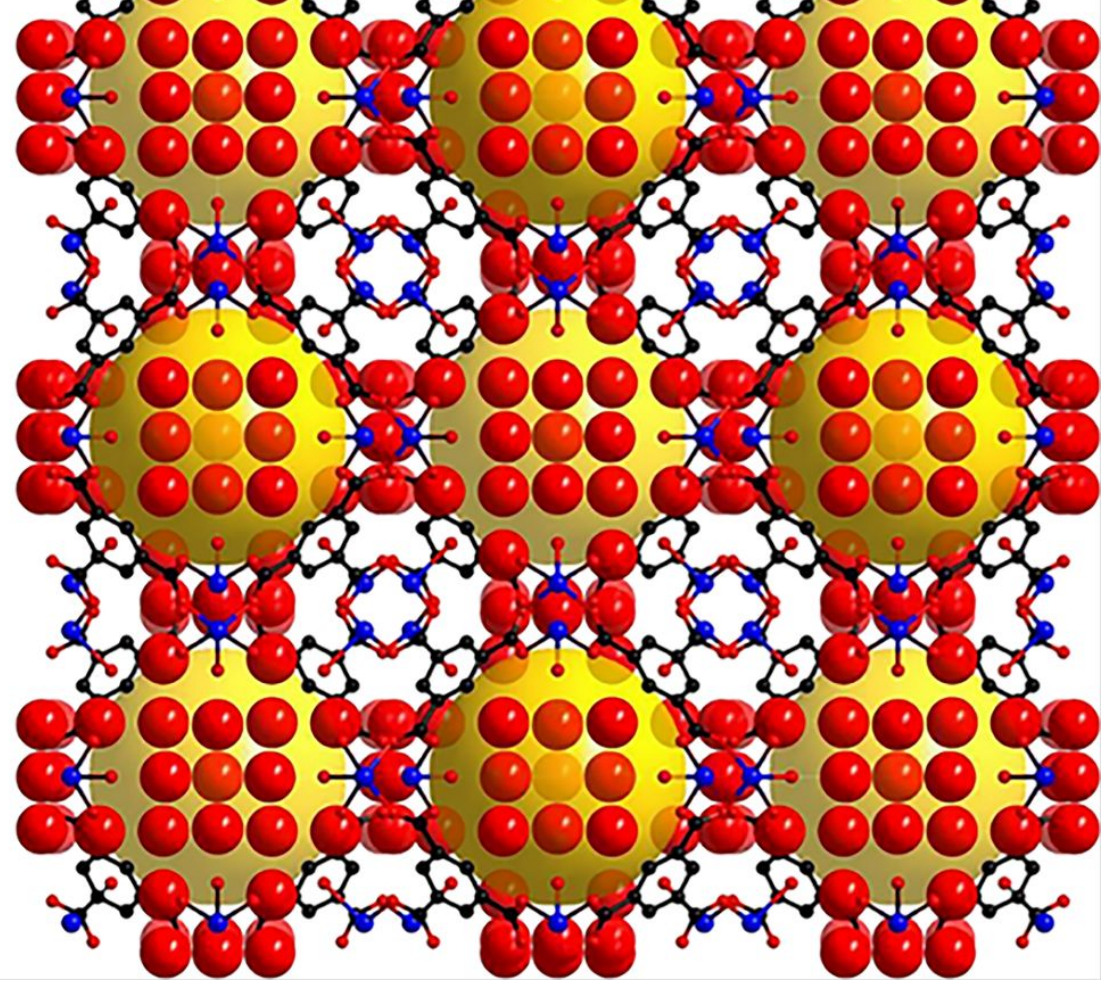

Figure S4. (a) The 3D structure of compound 1; (b) the 3D structure of compound 2. 

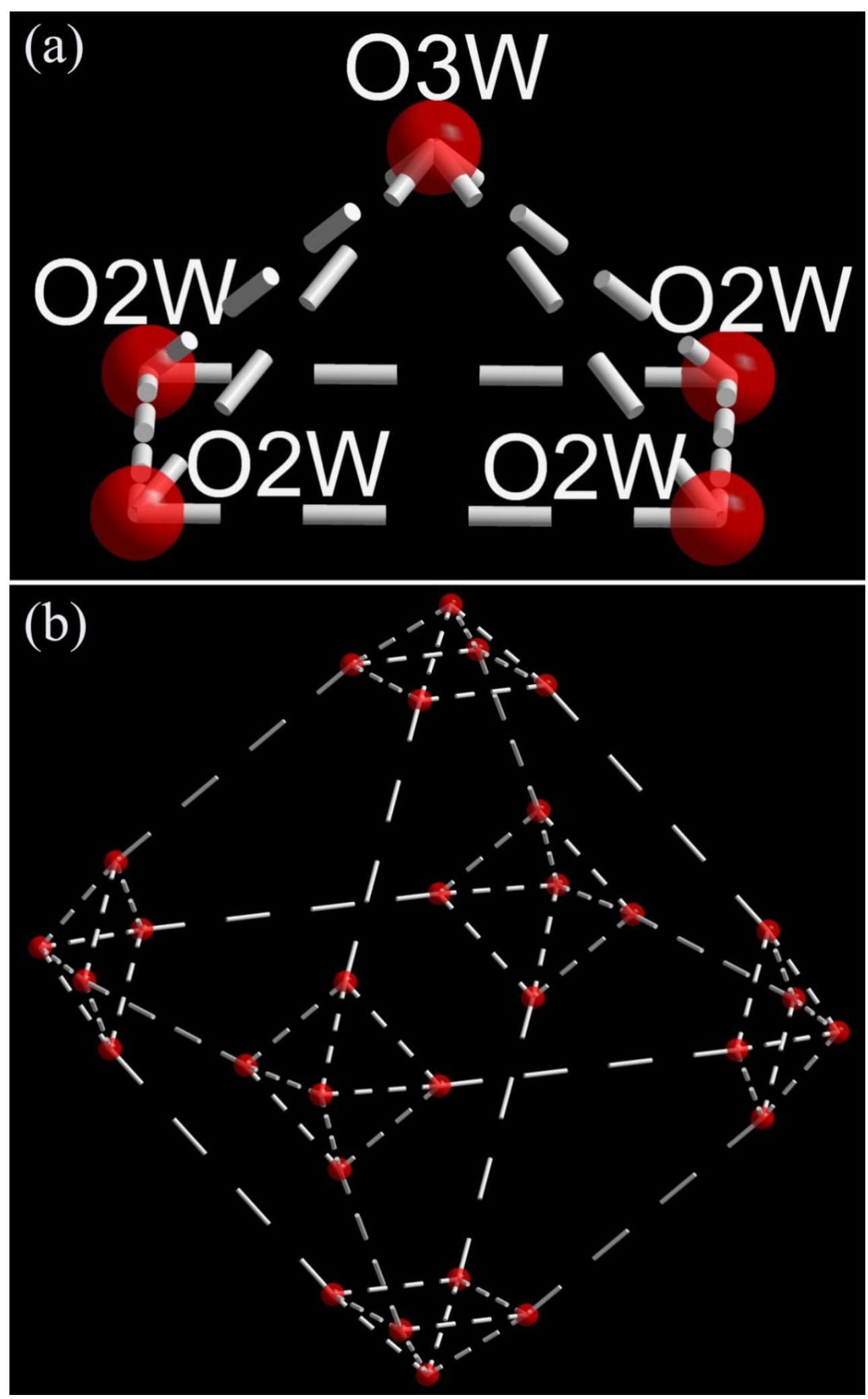

Figure S5. (a) The square-pyramid-shaped water cluster $\left(\mathrm{H}_{2} \mathrm{O}\right)_{5}$; (b) the configuration of the octahedral-liked water cluster $\left(\mathrm{H}_{2} \mathrm{O}\right)_{30}$. 


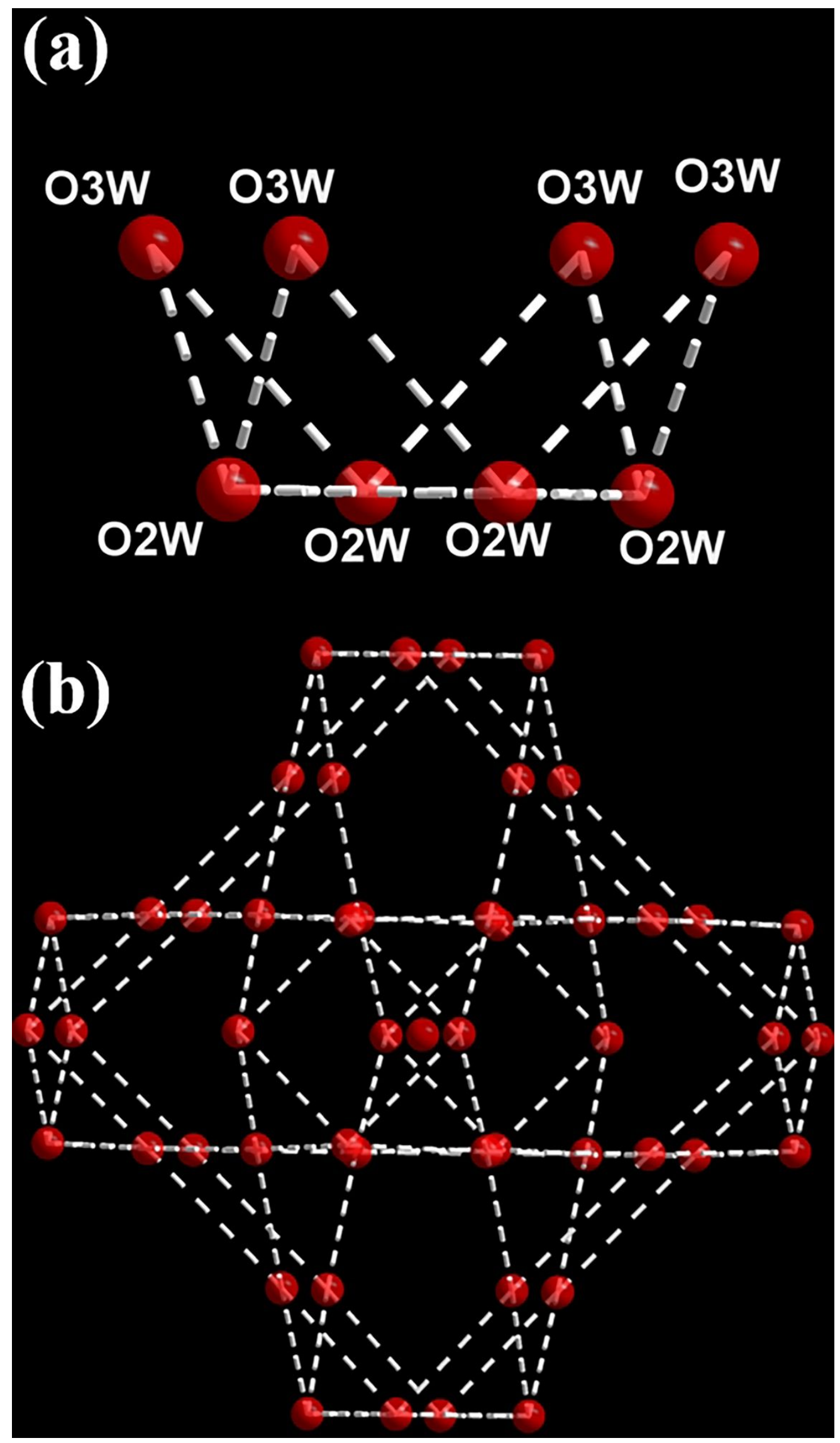

Figure S6. (a) The crown-like water cluster $\left(\mathrm{H}_{2} \mathrm{O}\right)_{8}$; (b) the configuration of the cage-shaped water cluster $\left(\mathrm{H}_{2} \mathrm{O}\right)_{49}$. 


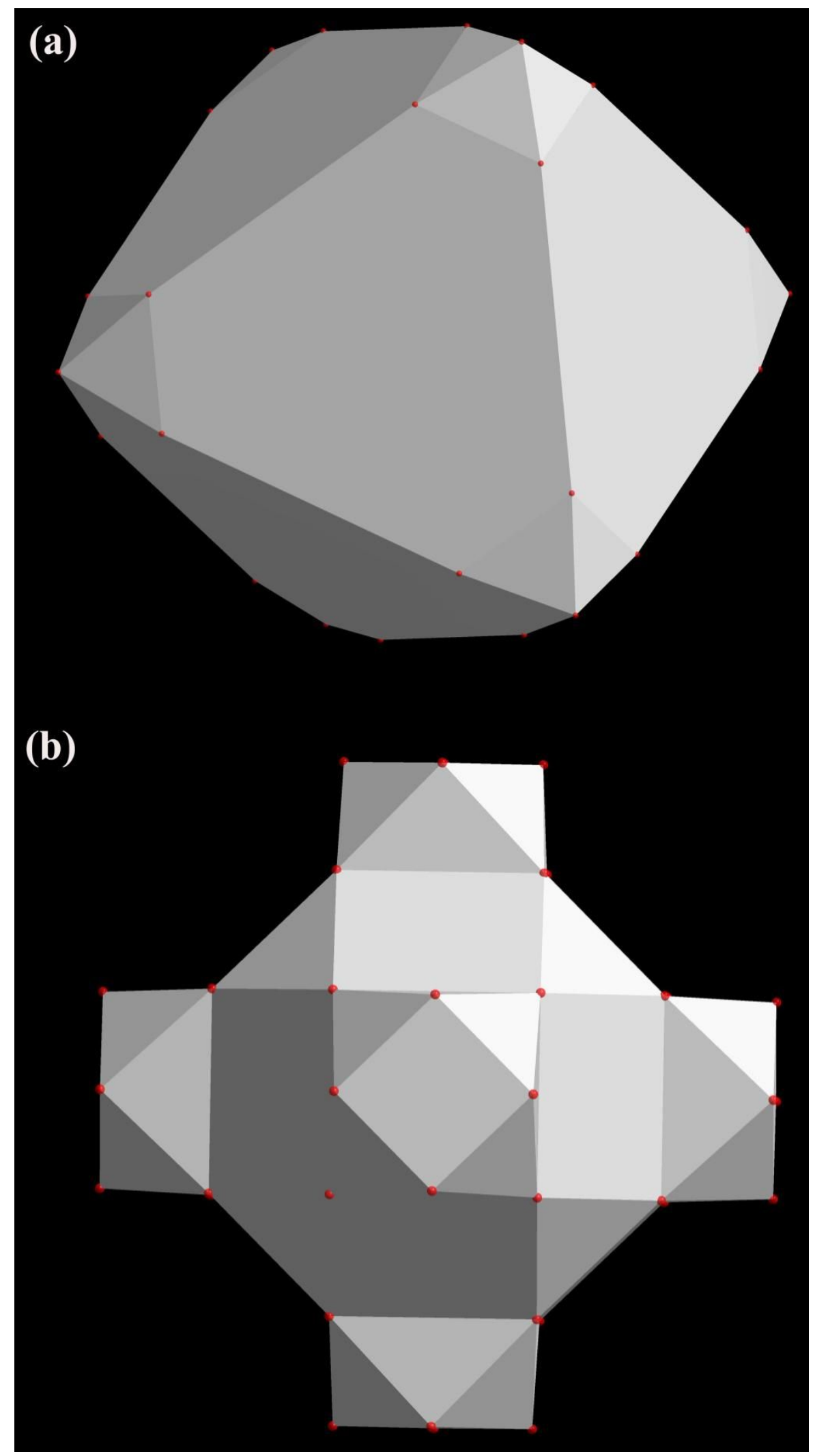

Figure S7. (a) The polyhedral representation of water cluster $\left(\mathrm{H}_{2} \mathrm{O}\right)_{30}$; (b) the polyhedral representation of water cluster $\left(\mathrm{H}_{2} \mathrm{O}\right)_{49}$. 
(a)

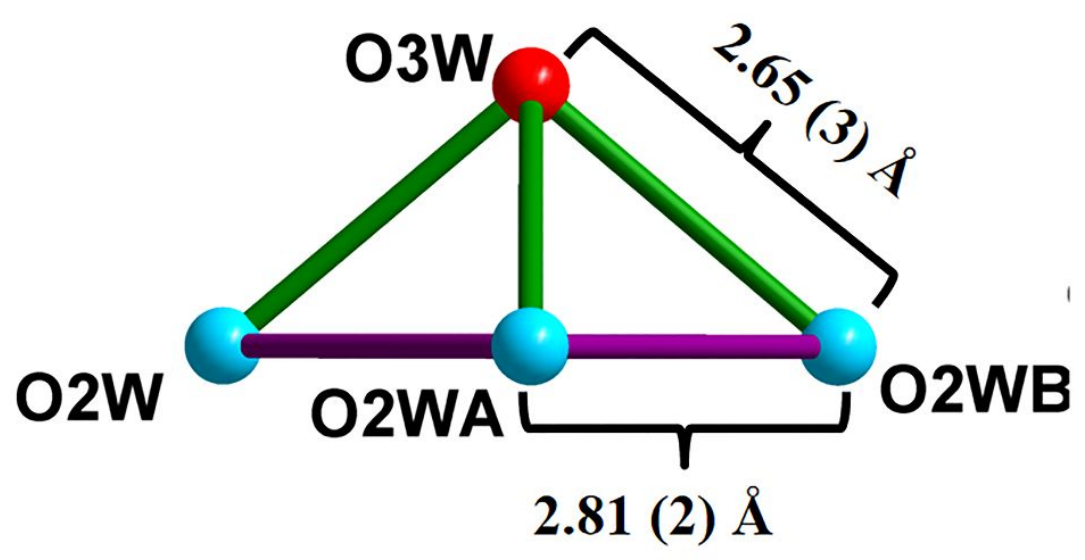

(b)

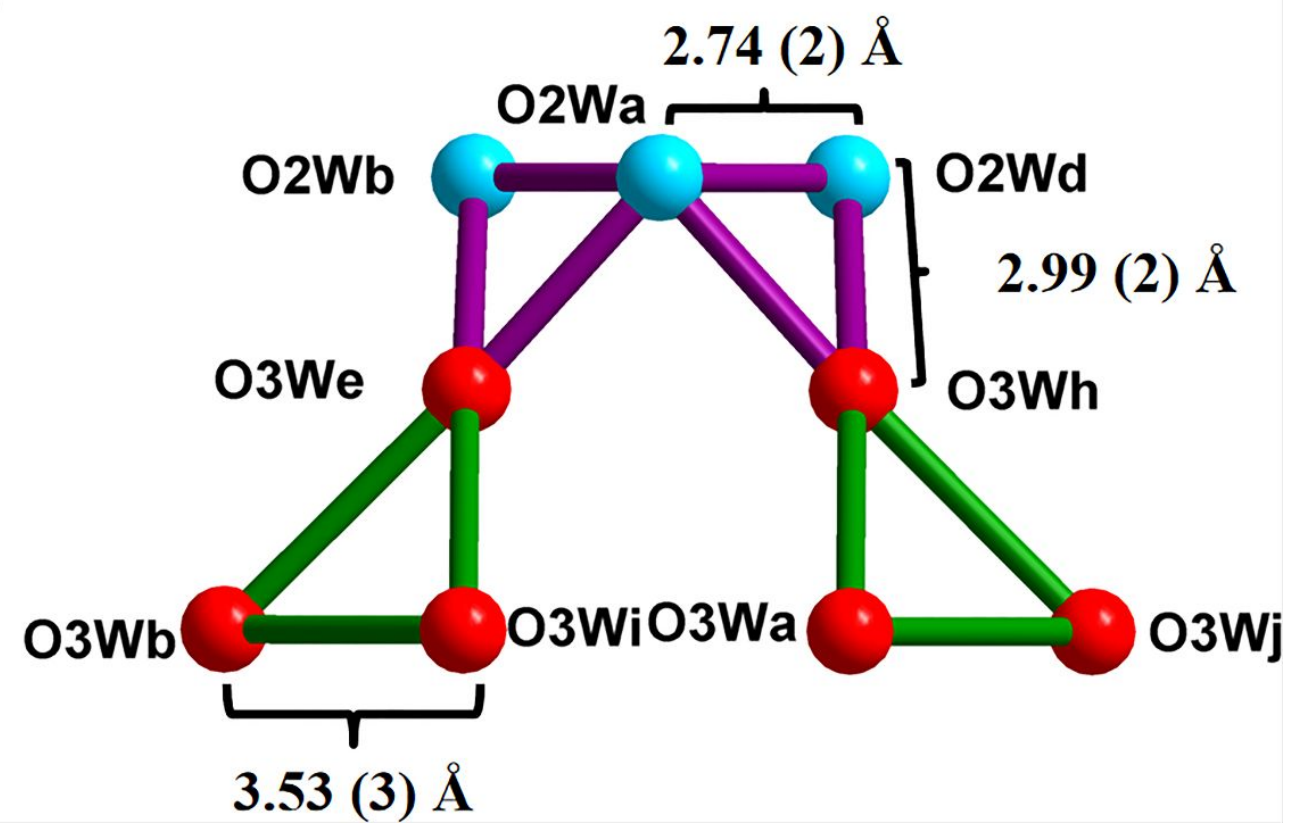

Figure S8. (a) The coordination environment of $\mathrm{O} 2 \mathrm{~W}$ and $\mathrm{O} 3 \mathrm{~W}$ in compound $\mathbf{1 .}$ Symmetry codes: A: $-\mathrm{z}, \mathrm{y}, \mathrm{x}$; B: $-\mathrm{x}, \mathrm{y},-\mathrm{z}$; (b) the coordination environment of $\mathrm{O} 2 \mathrm{~W}$ and $\mathrm{O} 3 \mathrm{~W}$ in compound 2 . Symmetry codes: a: $0.5+\mathrm{y}, \mathrm{z}, 0.5+\mathrm{x} ; \mathrm{b}: 0.5-\mathrm{x}, \mathrm{z}, 0.5+\mathrm{y}$; c: $0.5-y, z, 0.5-x ;$ d: $0.5+x, z, 0.5-y$; e: 0.5-y, x, 0.5+z; f: 0.5-z, x, 0.5-y; g: 0.5+y, x, 0.5-z; h: 0.5+z, x, 0.5+y, h: 0.5+z. x, 0.5+y, i: 0.5-z, y, 0.5+x, j: 0.5+x, y, 0.5+z. 
Section 2. Characterizations

IR
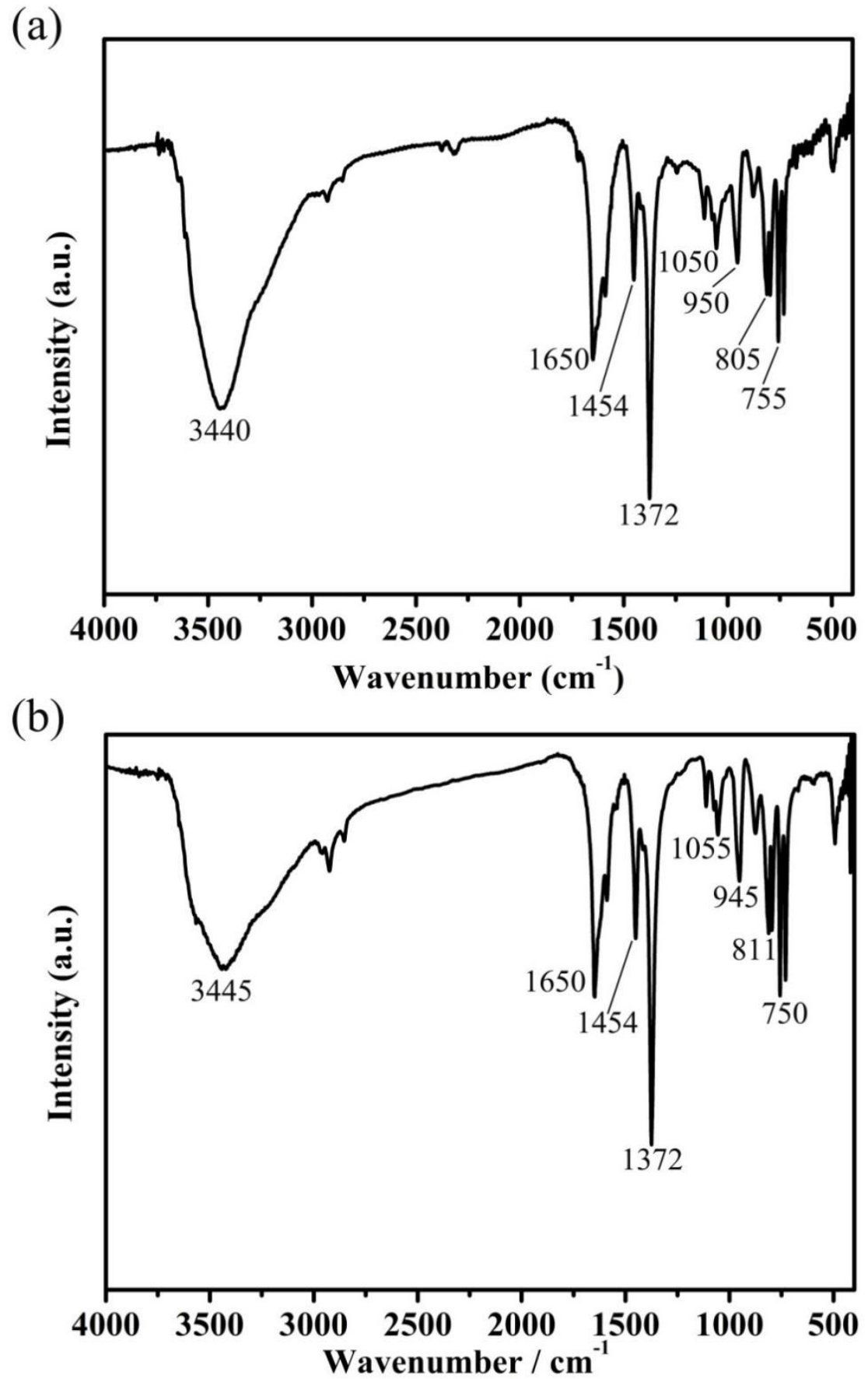

Figure S9. (a) The IR spectra of compound 1; (b) the IR spectra of compound 2. 
PXRD

(a)

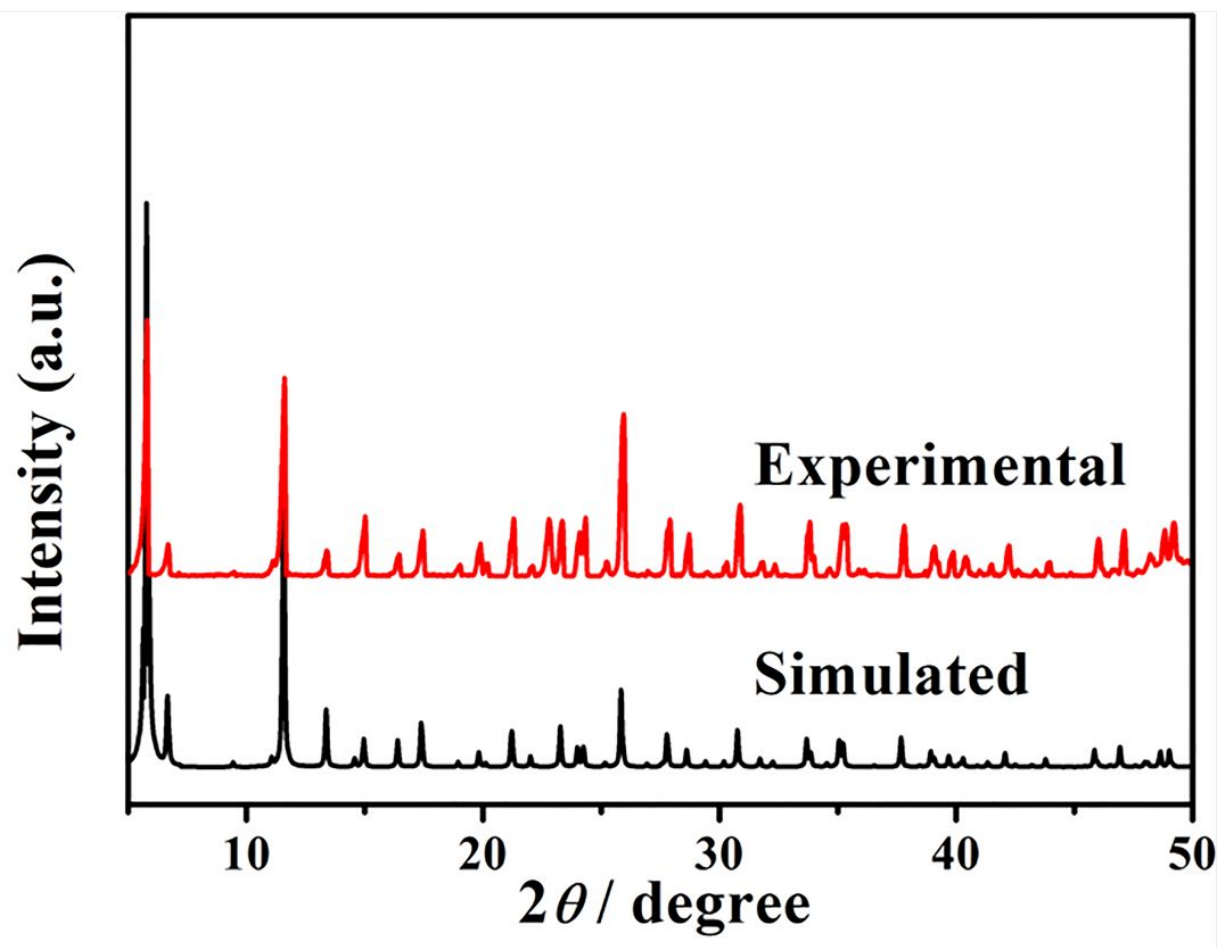

(b)

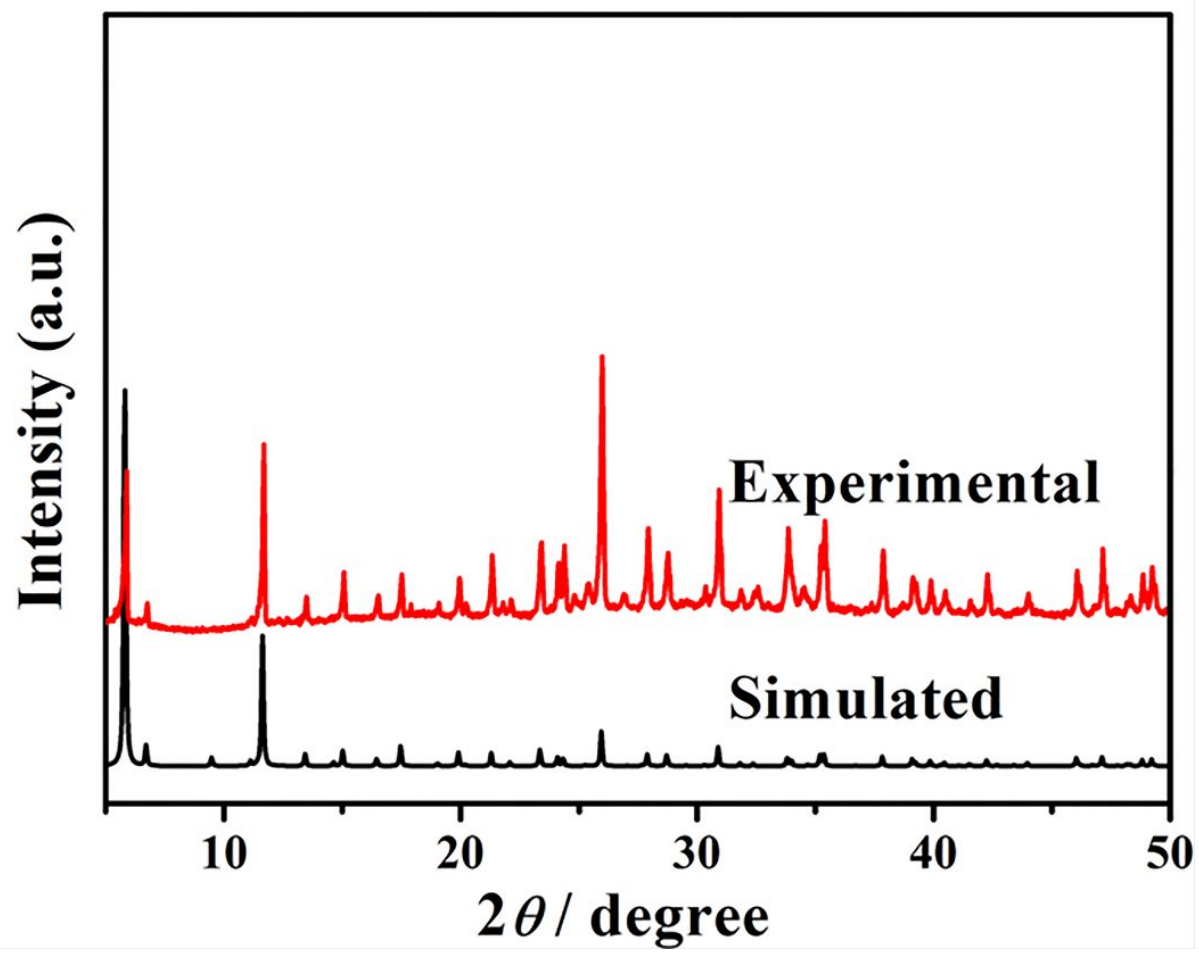

Figure S10. (a) The XRD pattern of compound 1; (b) the XRD pattern of compound 2. 
TGA

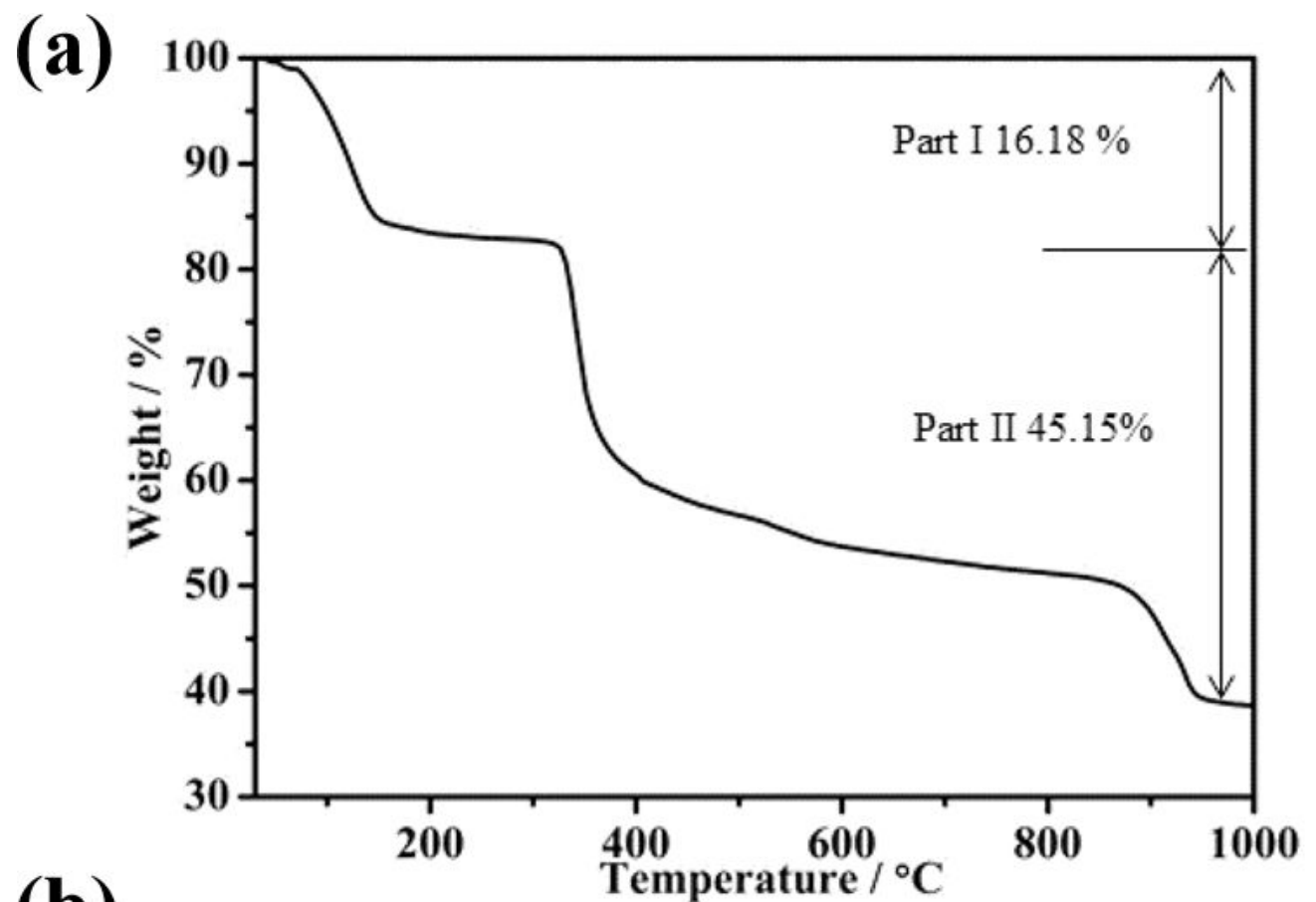

(b)

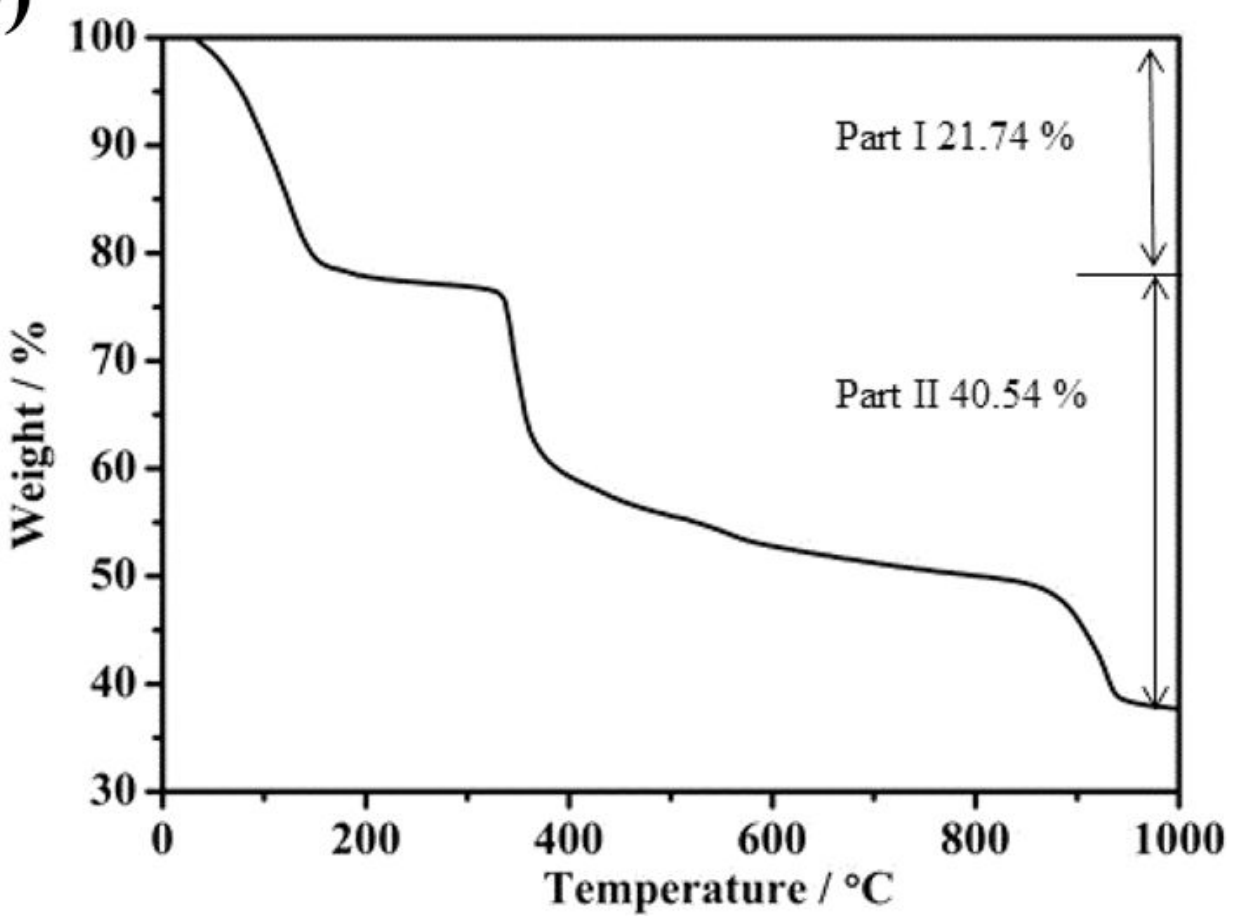

Figure S11. (a) The TG curve of compound 1; (b) the TG curve of compound 2.

TGA was performed $\left(\mathrm{T}=25-1000{ }^{\circ} \mathrm{C}\right)$ in air at a heating rate of $10{ }^{\circ} \mathrm{C} \mathrm{min}-1$ for the two compounds. As displayed in Figure. S9, the two compounds exist two similar continuous processes of weight loss. The weight loss of the first process between 25 to $180{ }^{\circ} \mathrm{C}$ is 16.18 and $21.74 \%$, respectively, (calc. 15.25 and $20.89 \%$ ), attributing to 
the loss of water clusters and 12 coordination water molecules in the two compounds. Between $180{ }^{\circ} \mathrm{C}$ and $1000{ }^{\circ} \mathrm{C}$, the weight loss of 45.15 and $40.54 \%$ is corresponding with assigned to the collapse of the $\mathrm{Cu}_{3}(\mathrm{BTC})_{2}$ and POMs. 


\section{SEM}

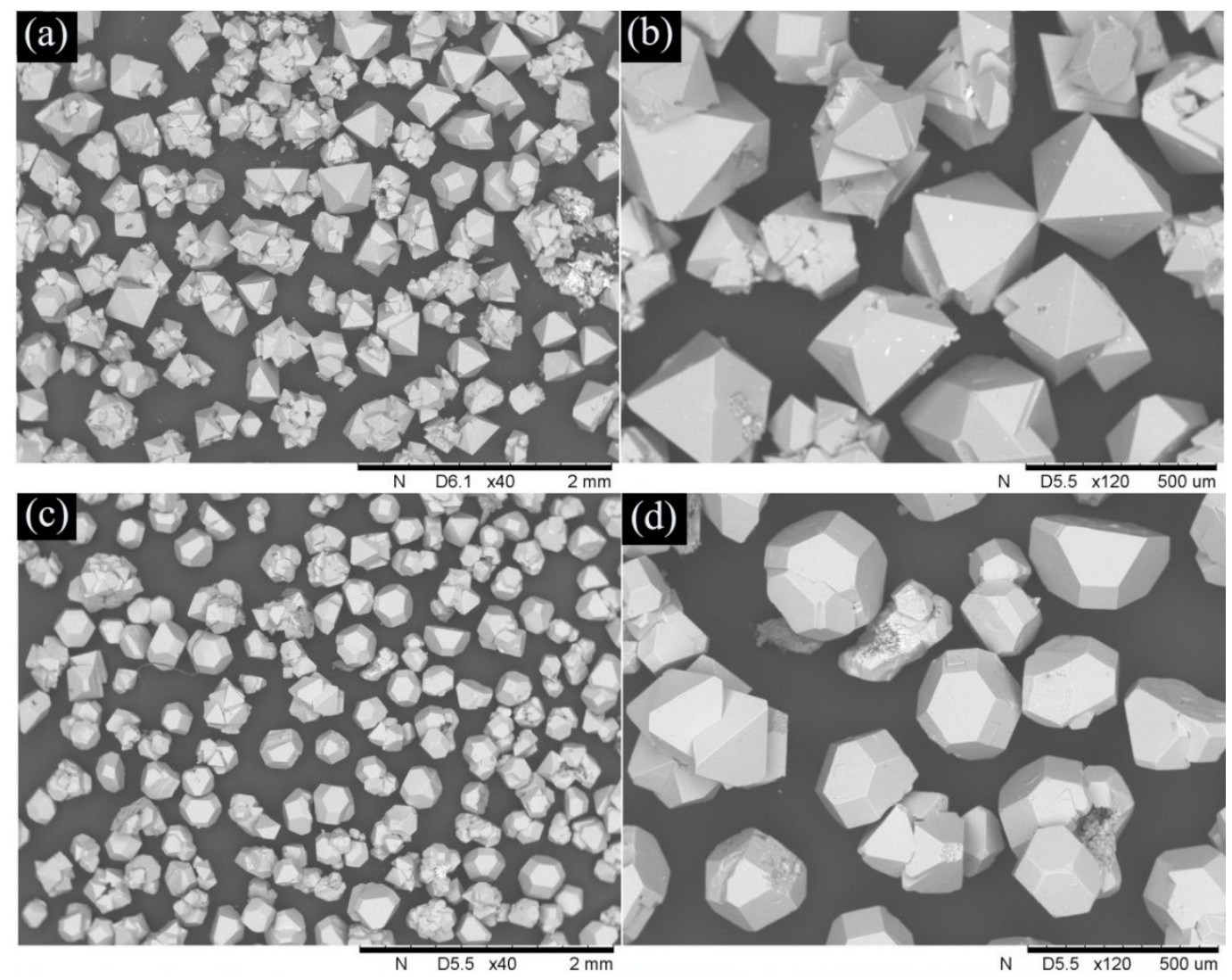

Figure S12. (a), (b) SEM images of compound 1; (c), (d) SEM images of compound 2.

\section{EDS mapping}

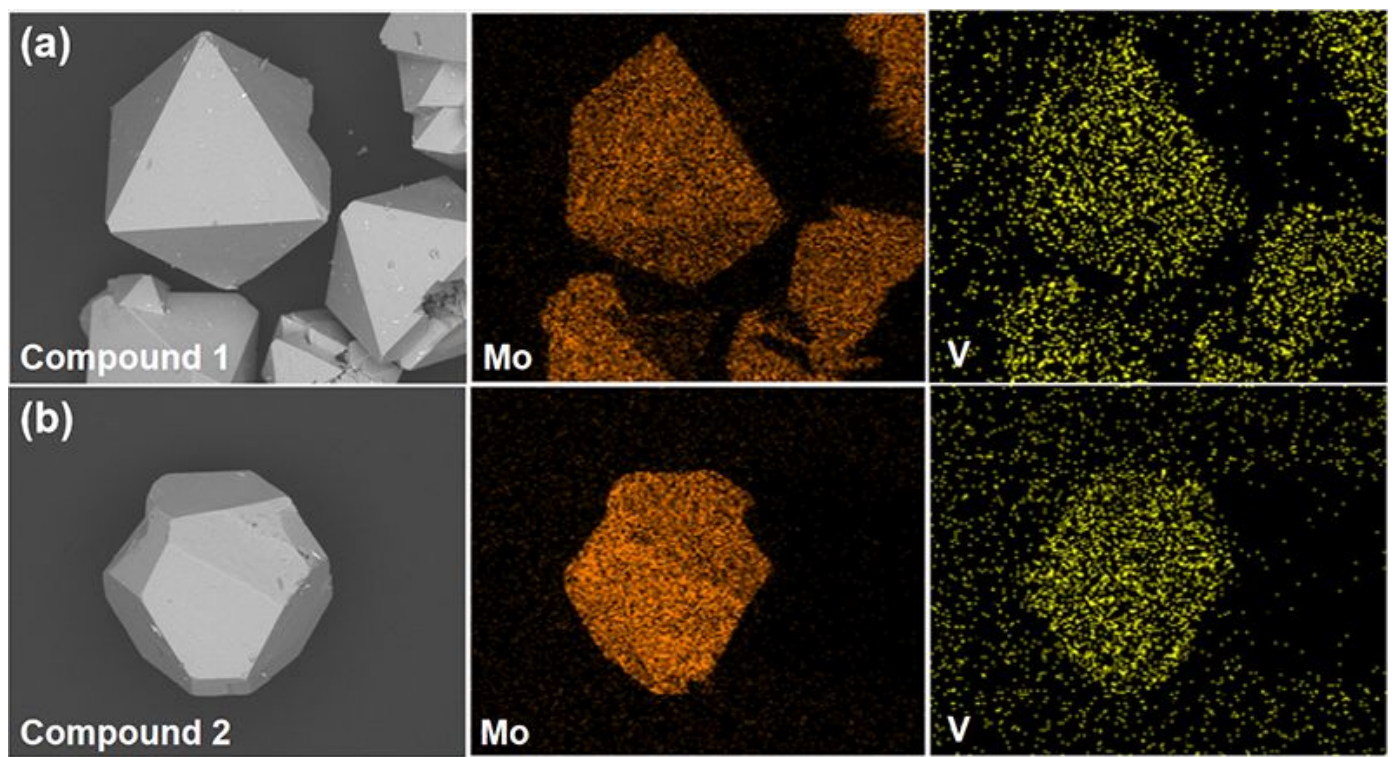

Figure S13. (a) EDS-mapping of compound 1; (b) EDS-mapping of compound 2. 


\section{EDS measurement}
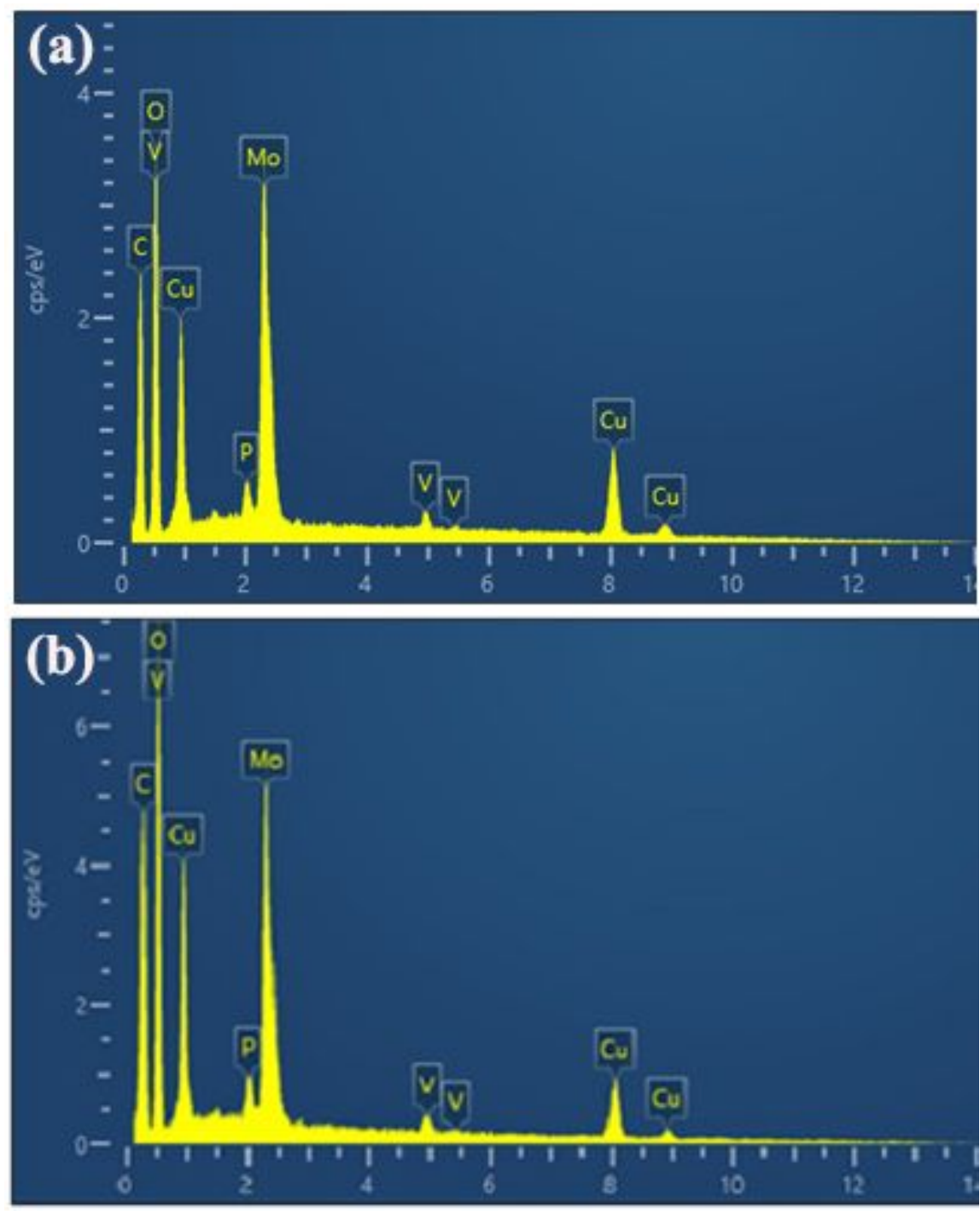

Figure S14. (a) The EDS measurement of compound 1; (b) the EDS measurement of compound 2 .

\section{ICP}

Table S1. The ICP result of the two compounds.

\begin{tabular}{|l|l|l|l|l|l|l|}
\hline \multicolumn{2}{|l|}{ Content } & $\begin{array}{l}\mathrm{Cu} \\
(\mathrm{mmol} / \mathrm{L})\end{array}$ & $\begin{array}{l}\mathrm{Mo} \\
(\mathrm{mmol} / \mathrm{L})\end{array}$ & $\begin{array}{l}\mathrm{V} \\
(\mathrm{mmol} / \mathrm{L})\end{array}$ & $\begin{array}{l}\mathrm{Cu}: \mathbf{V} \\
(\mathrm{mol} \%)\end{array}$ & $\begin{array}{l}\text { Mo:V } \\
(\mathrm{mol} \%)\end{array}$ \\
\hline \multirow{2}{*}{$\mathbf{1}$} & 1 & $7.1384 \times 10^{-2}$ & $6.5811 \times 10^{-2}$ & $0.5647 \times 10^{-2}$ & 12.64 & 11.65 \\
\cline { 2 - 7 } & 2 & $6.8963 \times 10^{-2}$ & $6.4695 \times 10^{-2}$ & $0.5685 \times 10^{-2}$ & 12.13 & 11.38 \\
\hline \multirow{2}{*}{$\mathbf{2}$} & 1 & $8.4625 \times 10^{-2}$ & $7.3009 \times 10^{-2}$ & $1.3014 \times 10^{-2}$ & 6.50 & 5.61 \\
\cline { 2 - 7 } & 2 & $8.2463 \times 10^{-2}$ & $6.8080 \times 10^{-2}$ & $1.2943 \times 10^{-2}$ & 6.37 & 5.26 \\
\hline
\end{tabular}

The result of ICP is consistent with the SCXRD result. 


\section{Section 3. Benzene Hydroxylation}

\section{The Procedure of the Catalysis for the Oxidation of Benzene}

The benzene $(1 \mathrm{~mL}), 30 \%$ hydrogen peroxide $(2 \mathrm{~mL})$, catalyst $(0.03 \mathrm{~g})$ acetonitrile $(5 \mathrm{~mL})$ were added into $50 \mathrm{~mL}$ three-necked flask equipped with a magnetic stirring rotor and reflux condenser. Then, reacting the mixture for 4 hours in $65{ }^{\circ} \mathrm{C}$ water bath under stirring. 1,4-dioxane was used as internal standard, conversion and selectivity were calculated by gas chromatography analysis. Under this condition, in addition to phenol as main product, benzoquinone was detected as byproduct.

To remove water cluster, the catalysts were dried under $180{ }^{\circ} \mathrm{C}$ for 15 hours. During the recyclability experiments, catalysts were washed with ethanol.

Reusability and stability are important indicators to evaluate catalyst, after each reaction, the catalyst was recovered by centrifugation and washed several times with $\mathrm{C}_{2} \mathrm{H}_{5} \mathrm{OH}$ and then dried at $80{ }^{\circ} \mathrm{C}$ for $12 \mathrm{~h}$ before the next reaction.

The conversion and selectivity are calculated by following ways:

Conversion $=\frac{\mathrm{n}_{2}+\mathrm{n}_{3}}{\mathrm{n}_{1}} \times 100 \% ；$

Phenol selectivity $=\frac{\mathrm{n}_{2}}{\mathrm{n}_{2}+\mathrm{n}_{3}} \times 100 \%$;

Phenol yield $=$ Conversion $\times$ Selectivity

Note: $\mathrm{n}_{1}=$ amount of benzene added, $\mathrm{n}_{2}=$ amount of phenol formed, $\mathrm{n}_{3}=$ amount of benzoquinone formed 


\section{Hot Filtration Test}

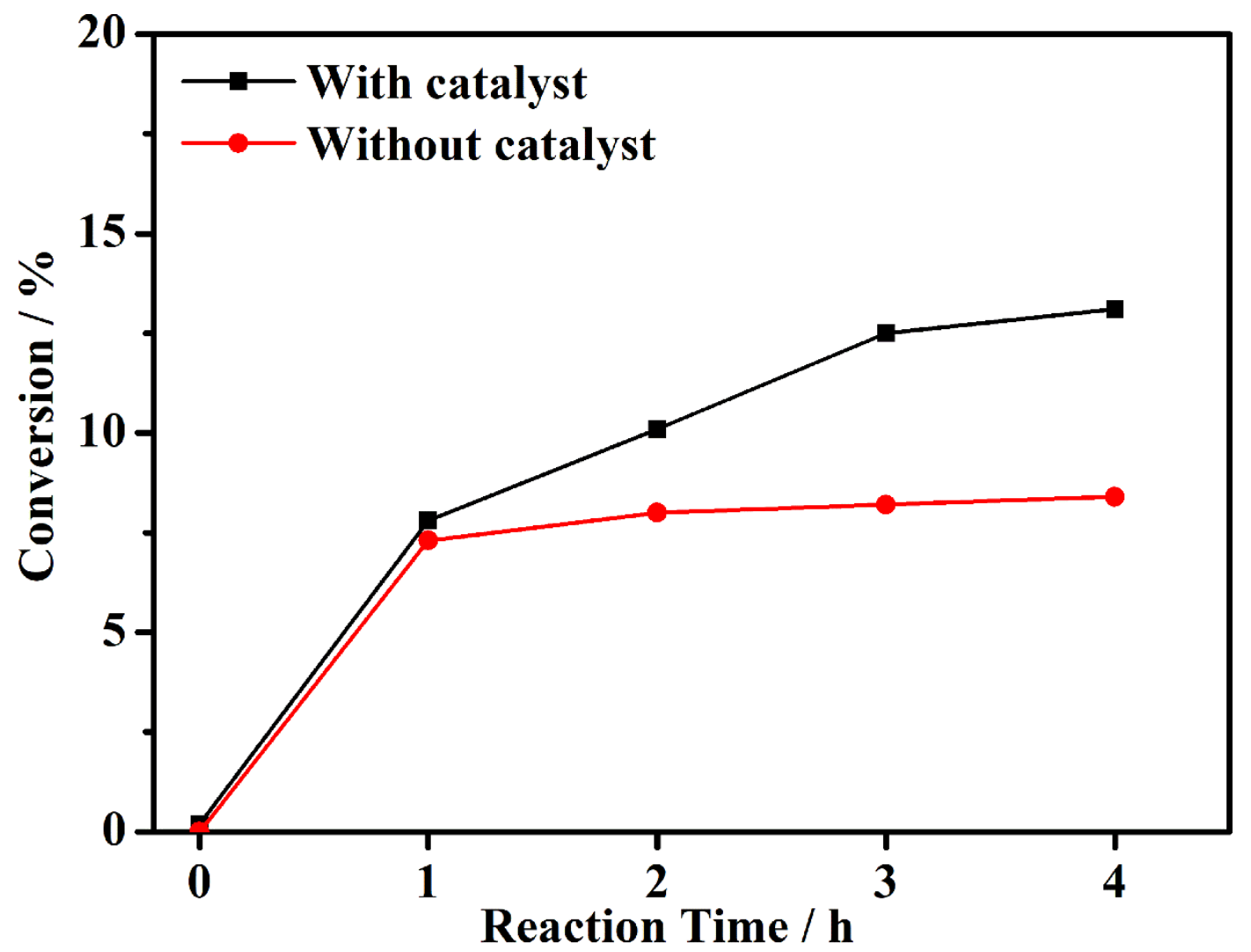

Figure S15. Hot filtration test for the compound $\mathbf{1}$ as catalyst in benzene hydroxylation. Red: filter out the catalyst during the reaction. Black: compound $\mathbf{1}$ as catalyst during the reaction. 
Recyclability Test

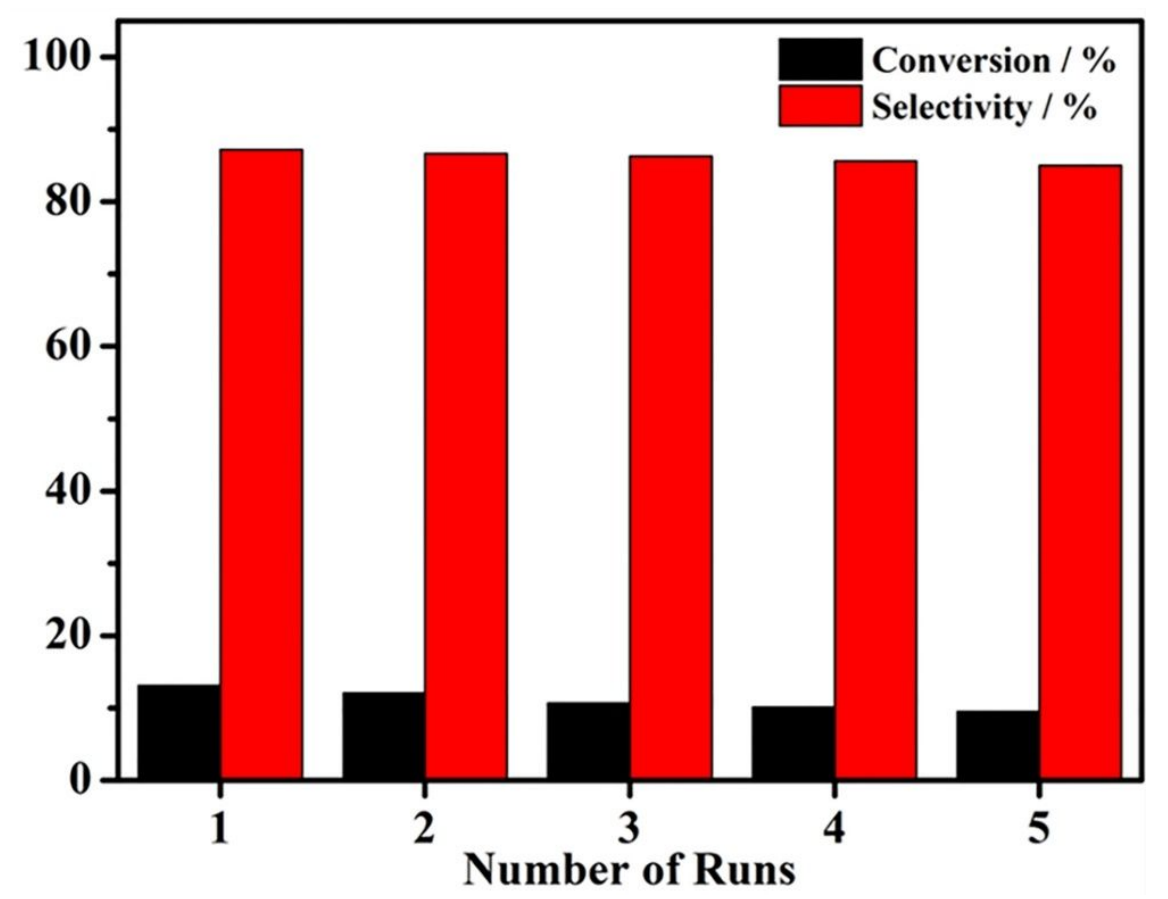

Figure S16. Recyclability of compound $\mathbf{1}$ as catalyst for benzene hydroxylation.

IR

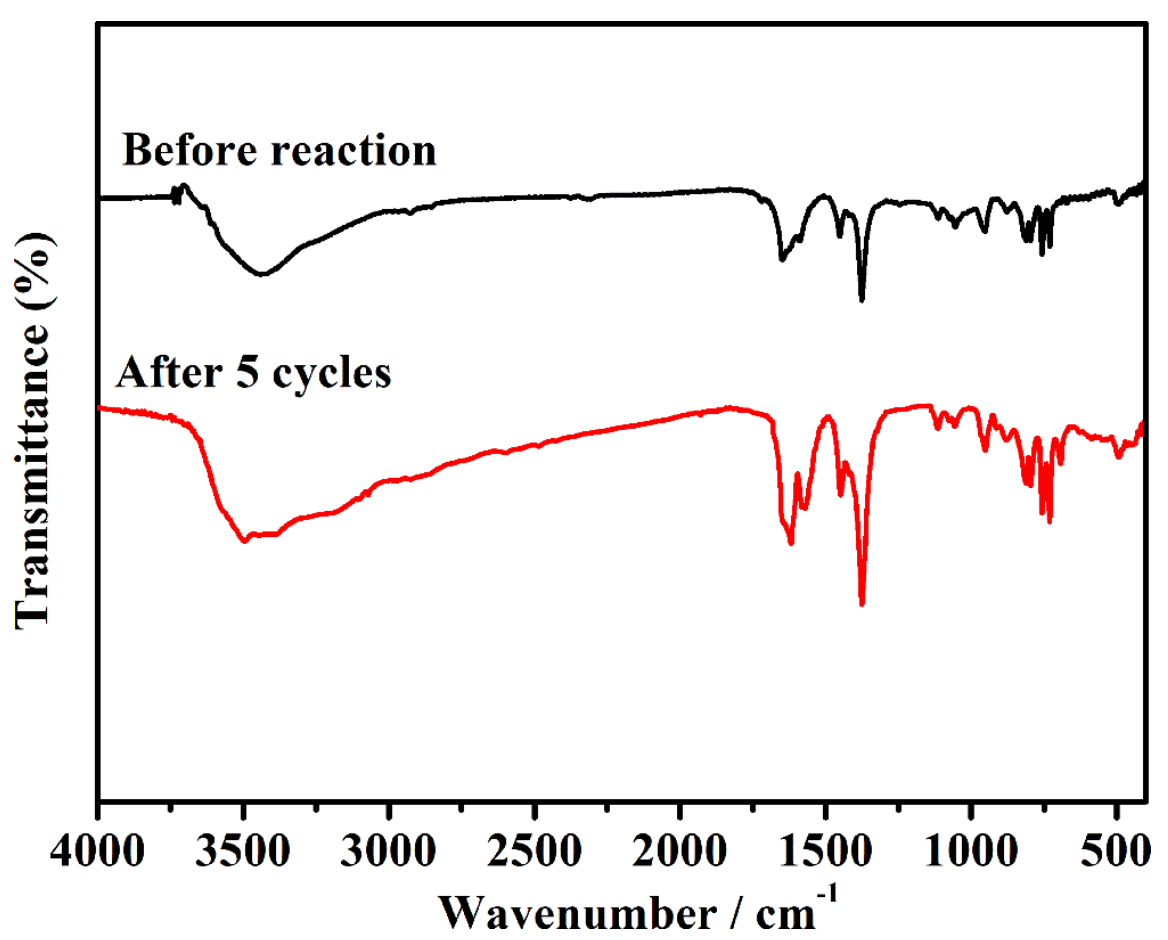

Figure S17. FTIR spectra of compound 1 before and after recycling for 5 times. 
XRD
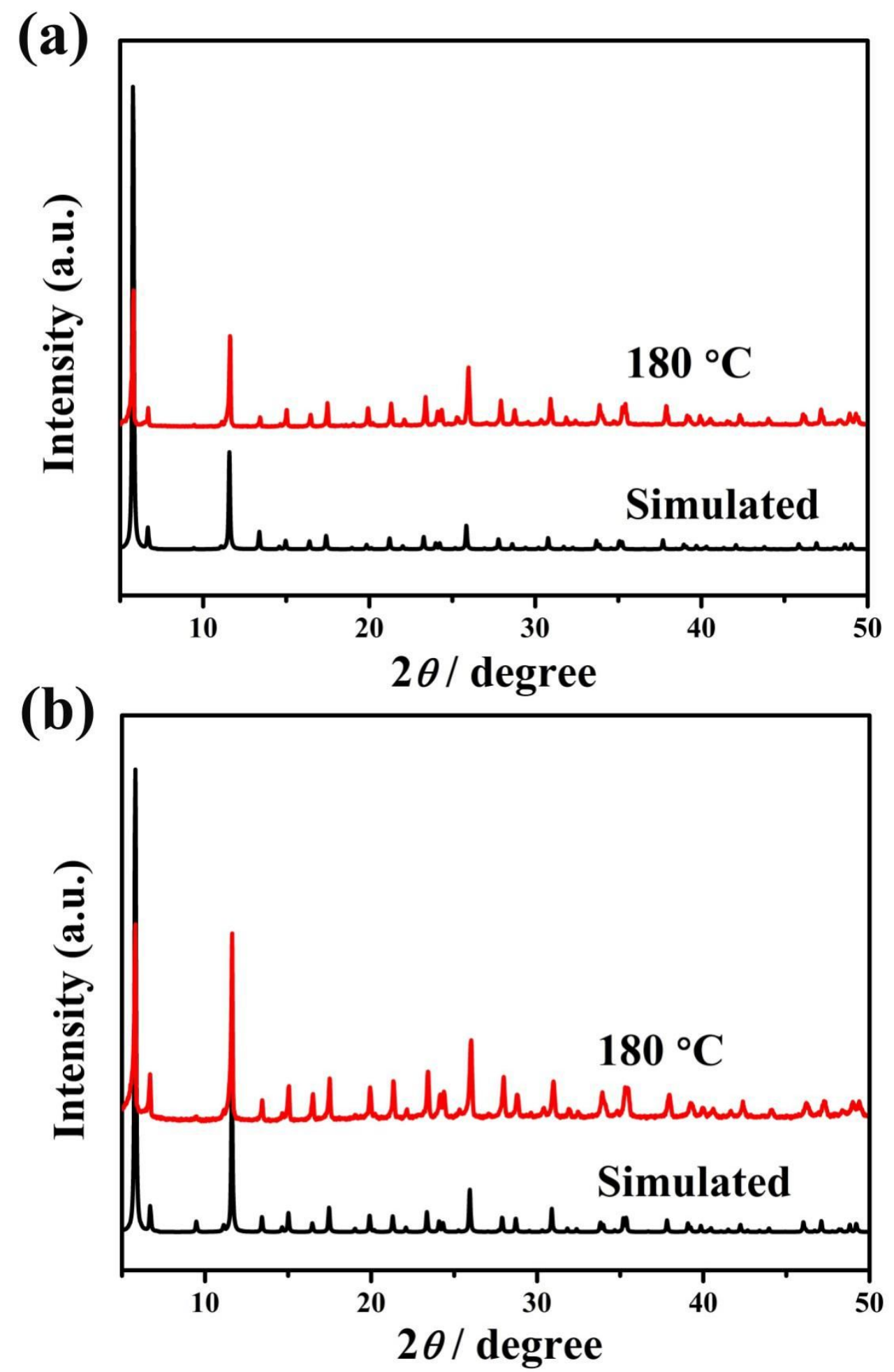

Figure S18. (a) The XRD pattern of compound 1 after heat treatment; (b) the XRD pattern of compound $\mathbf{2}$ after heat treatment. 


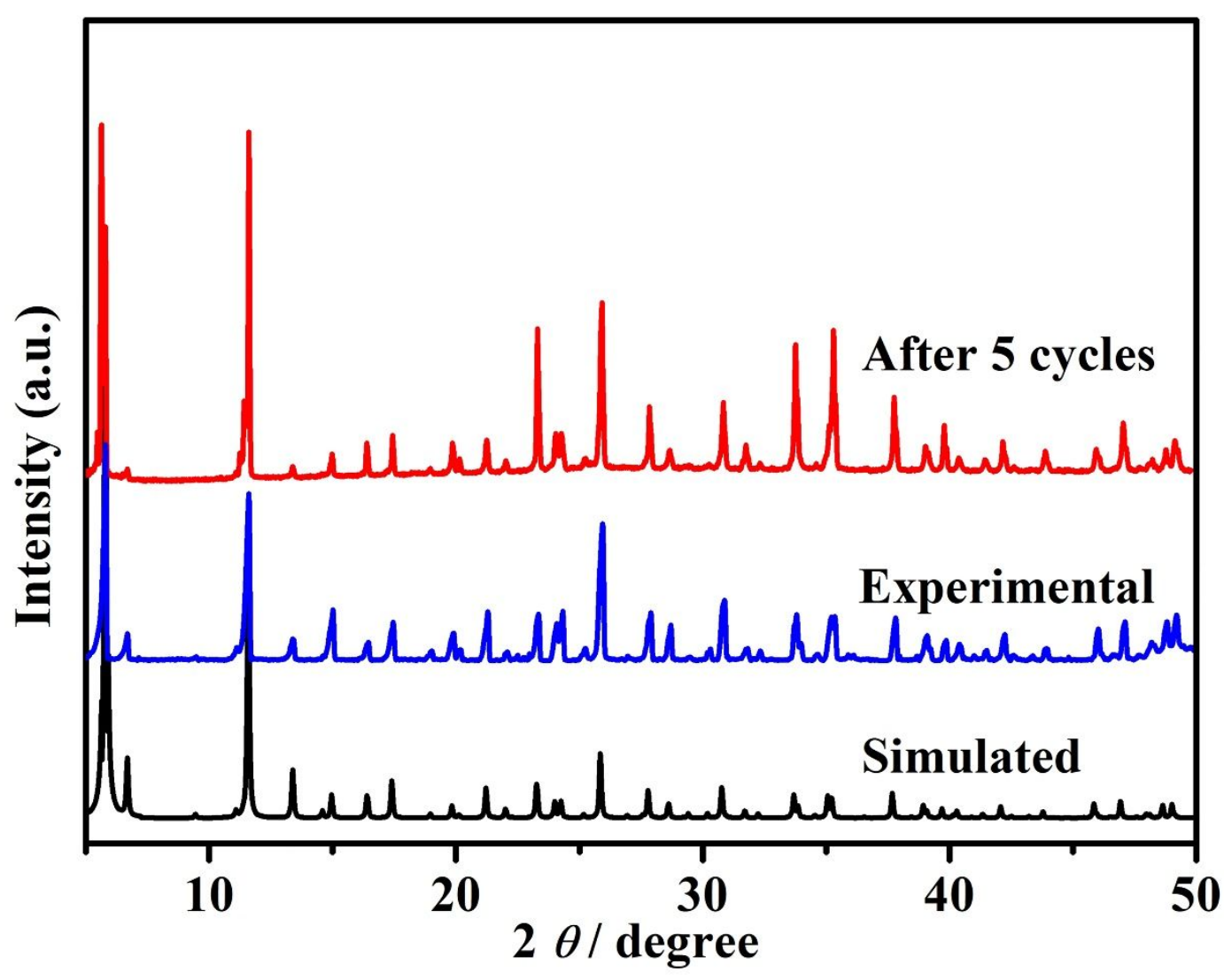

Figure S19. The XRD patterns of compound 1 after benzene hydroxylation.

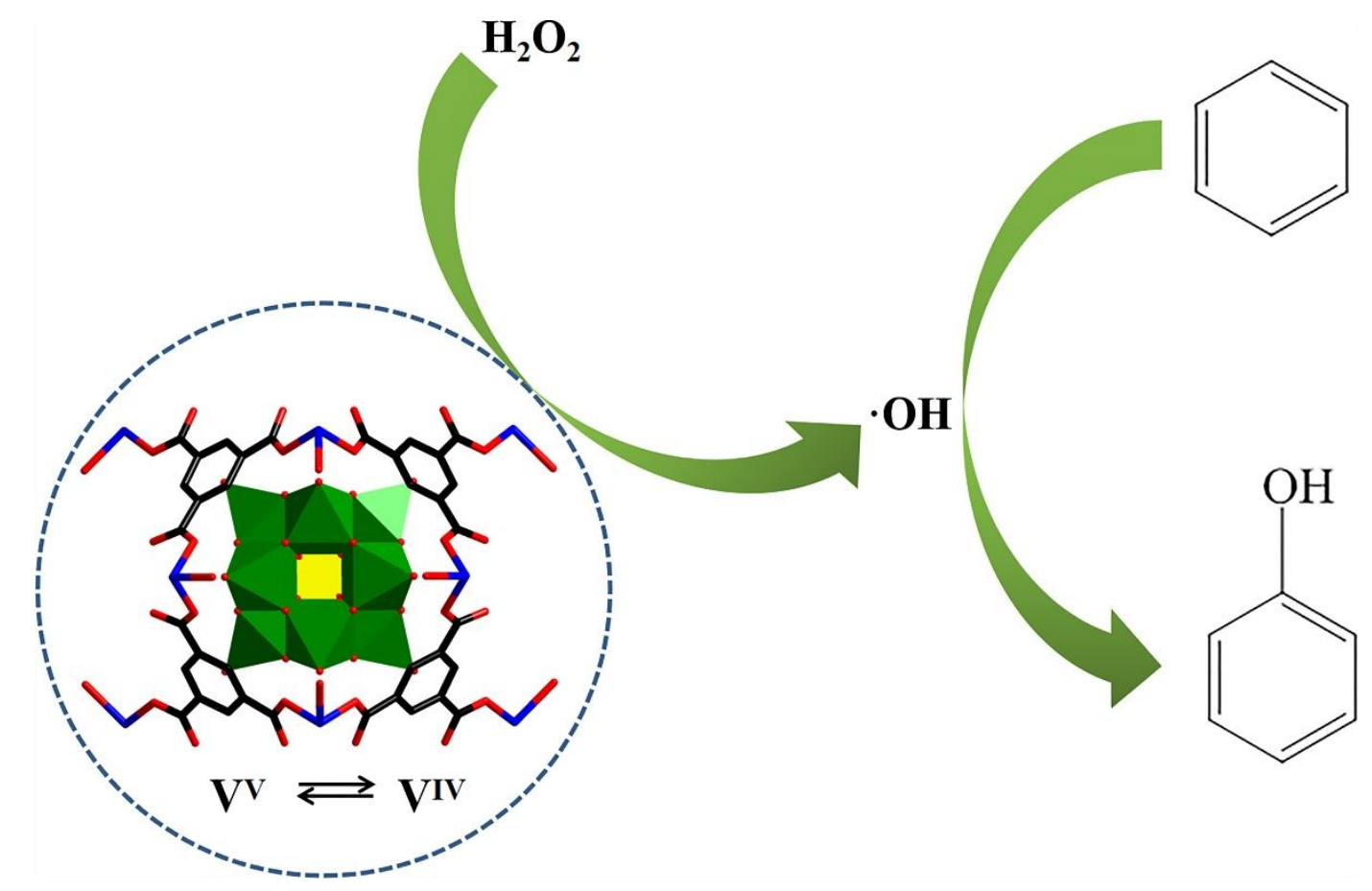

Figure S20. Proposed mechanism for the benzene ring catalyzed by compound $\mathbf{2}$ after heat treatment. 


\section{Section 4. Oxidative Desulfurization}

\section{The Procedure of the Catalysis for the Oxidative Desulfurization}

The substrate $(0.5 \mathrm{mmol})$, tert-Butyl hydroperoxide (TBHP) as oxidant, catalyst $(0.03 \mathrm{~g})$ and dichloromethane $(5 \mathrm{~mL})$ were added into $50 \mathrm{~mL}$ three-necked flask equipped with a magnetic stirring rotor and reflux condenser. Then, reacting the mixture for 5 hours in $65{ }^{\circ} \mathrm{C}$ water bath under stirring. n-dodecane was used as internal standard, the values of conversion and selectivity were calculated by gas chromatography analysis.

To remove water cluster, the catalysts were dried under $180{ }^{\circ} \mathrm{C}$ for 15 hours. During the recyclability experiments, catalysts were washed with ethanol.

$$
\text { Conversion }=\frac{\mathrm{n}_{2}}{\mathrm{n}_{1}} \times 100 \% ;
$$

Note: $\mathrm{n}_{1}=$ amount of substrate added, $\mathrm{n}_{2}=$ amount of product formed 


\section{Chemical Stability}

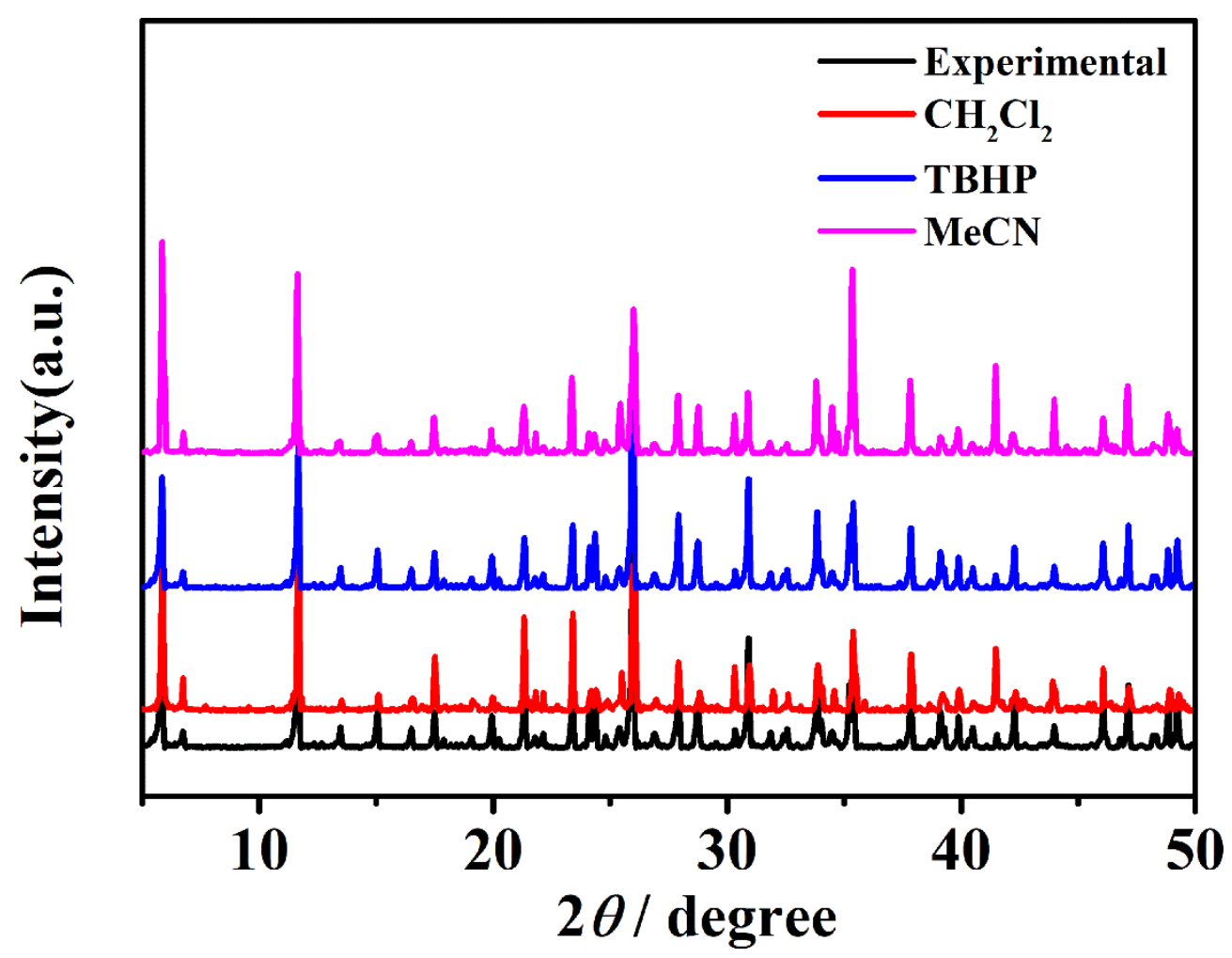

Figure S21. The XRD pattern of compound $\mathbf{2}$ in different solution.

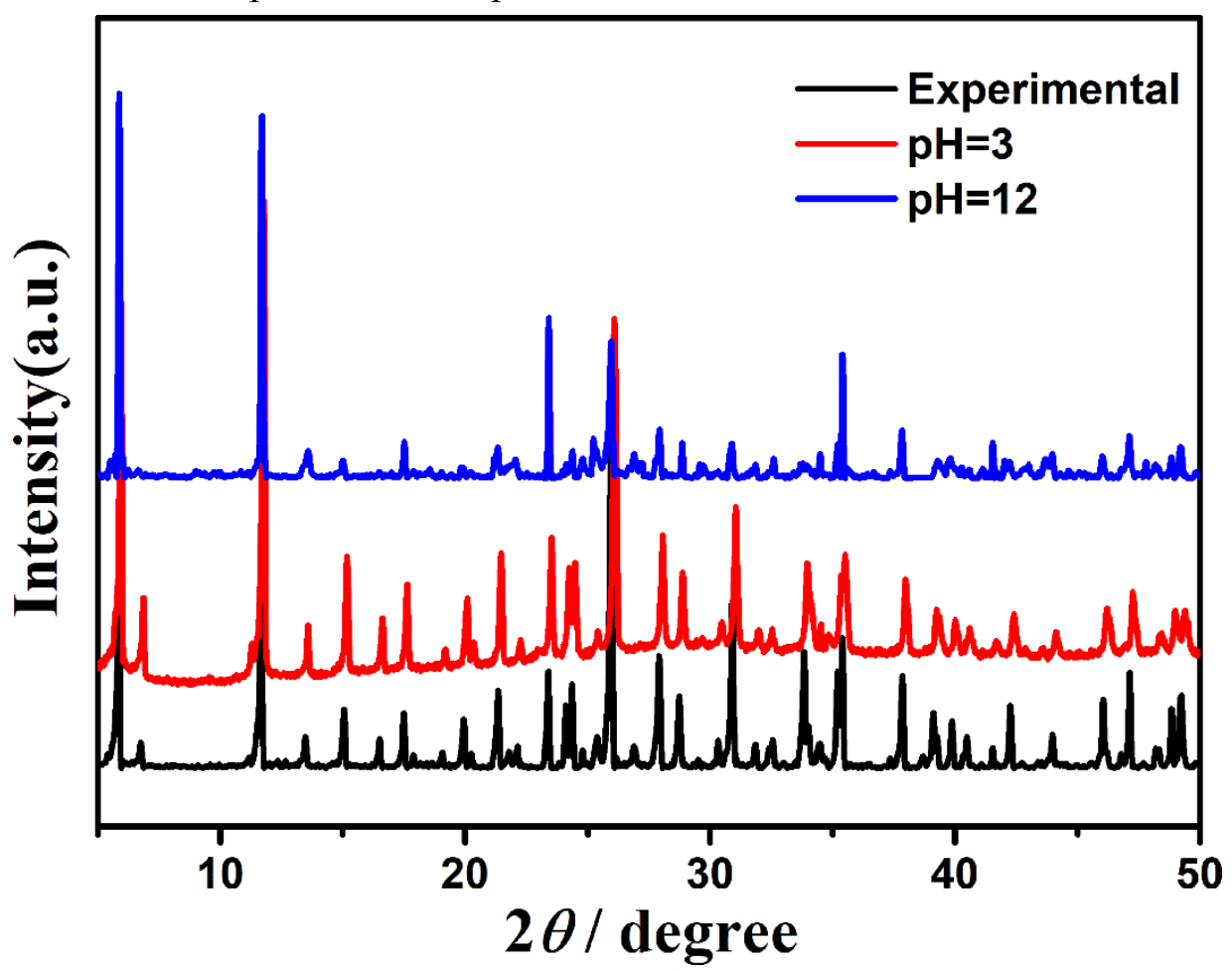

Figure S22. The XRD pattern of compound $\mathbf{2}$ in different $\mathrm{pH}$ values. 


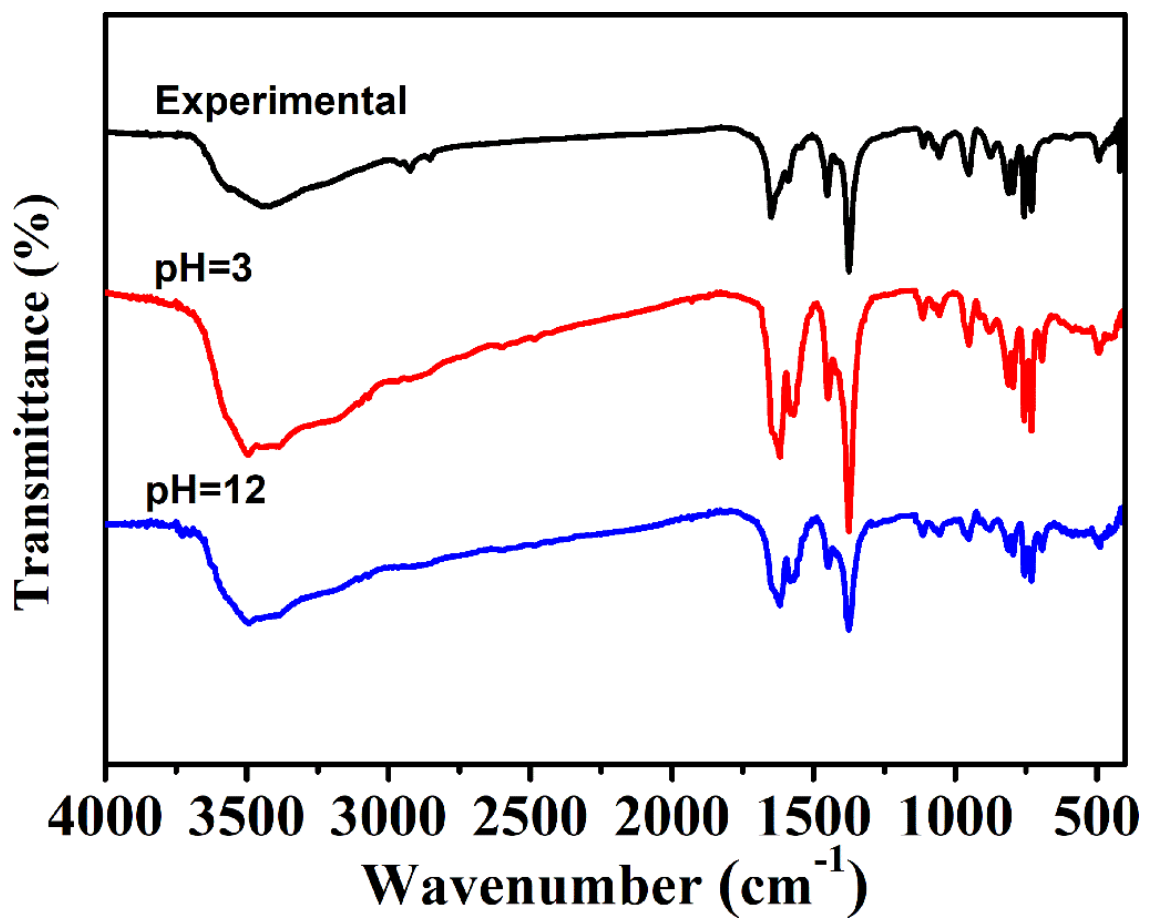

Figure S23. The IR spectra of compound $\mathbf{2}$ in different $\mathrm{pH}$ values.

The Investigation of Optimal Reaction Condition.

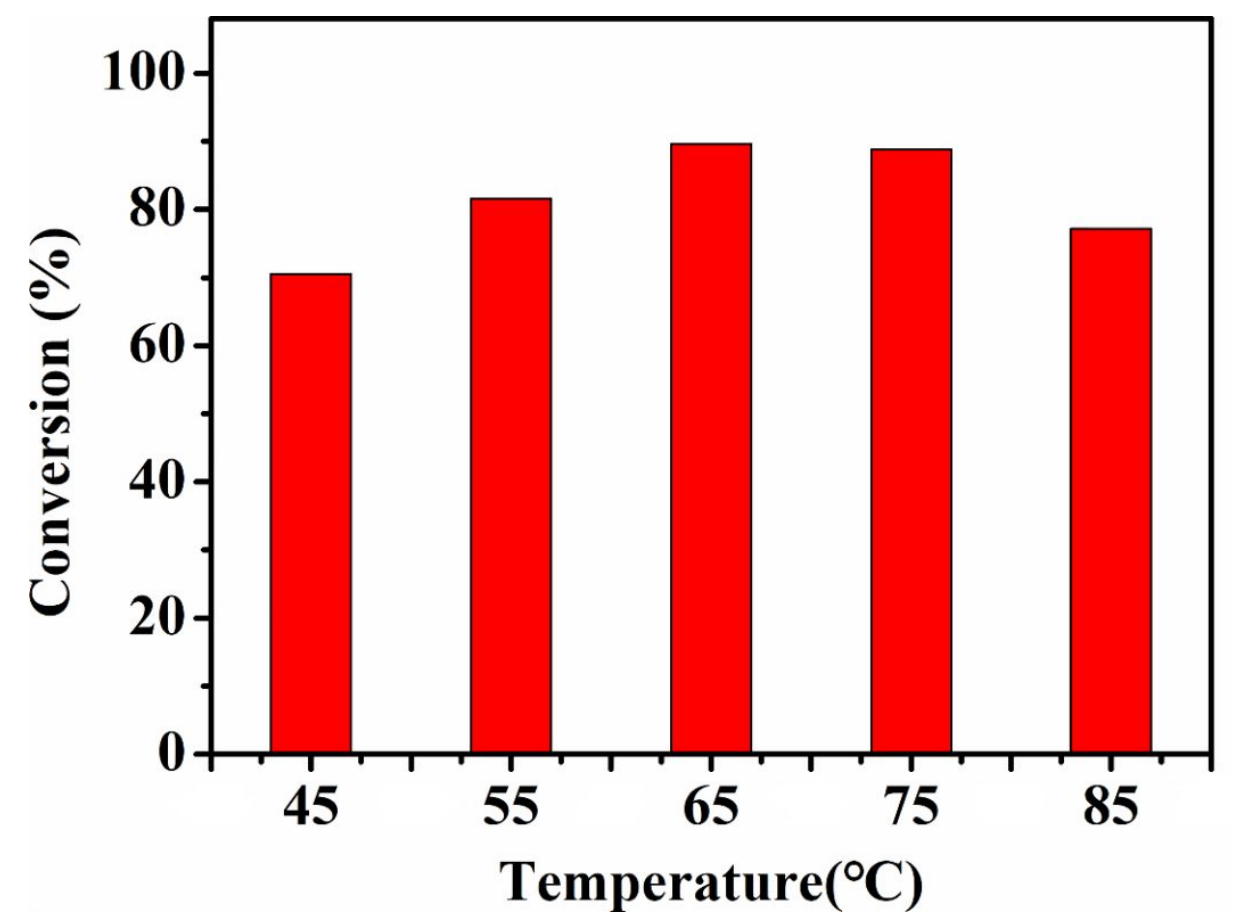

Figure S24. DBT conversion percent verse reaction temperature. Reaction condition: time $=5 \mathrm{~h}, \mathrm{O} / \mathrm{S}=6$. 


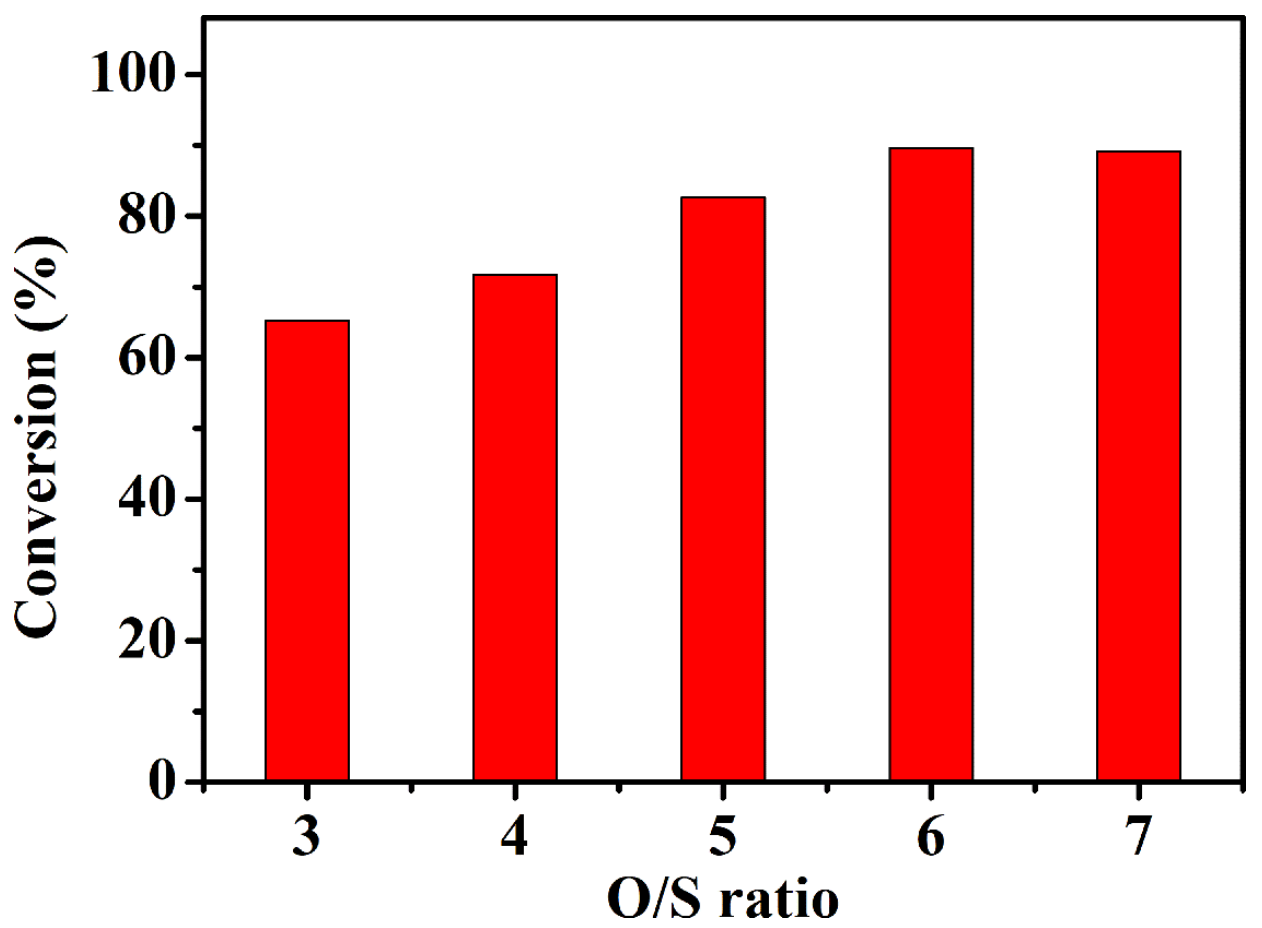

Figure S25. DBT conversion percent verse $\mathrm{O} / \mathrm{S}$ ratio. Reaction condition: time $=5 \mathrm{~h}$, $\mathrm{T}=65^{\circ} \mathrm{C}$.

Stability Test for the Compound 2 as Catalyst

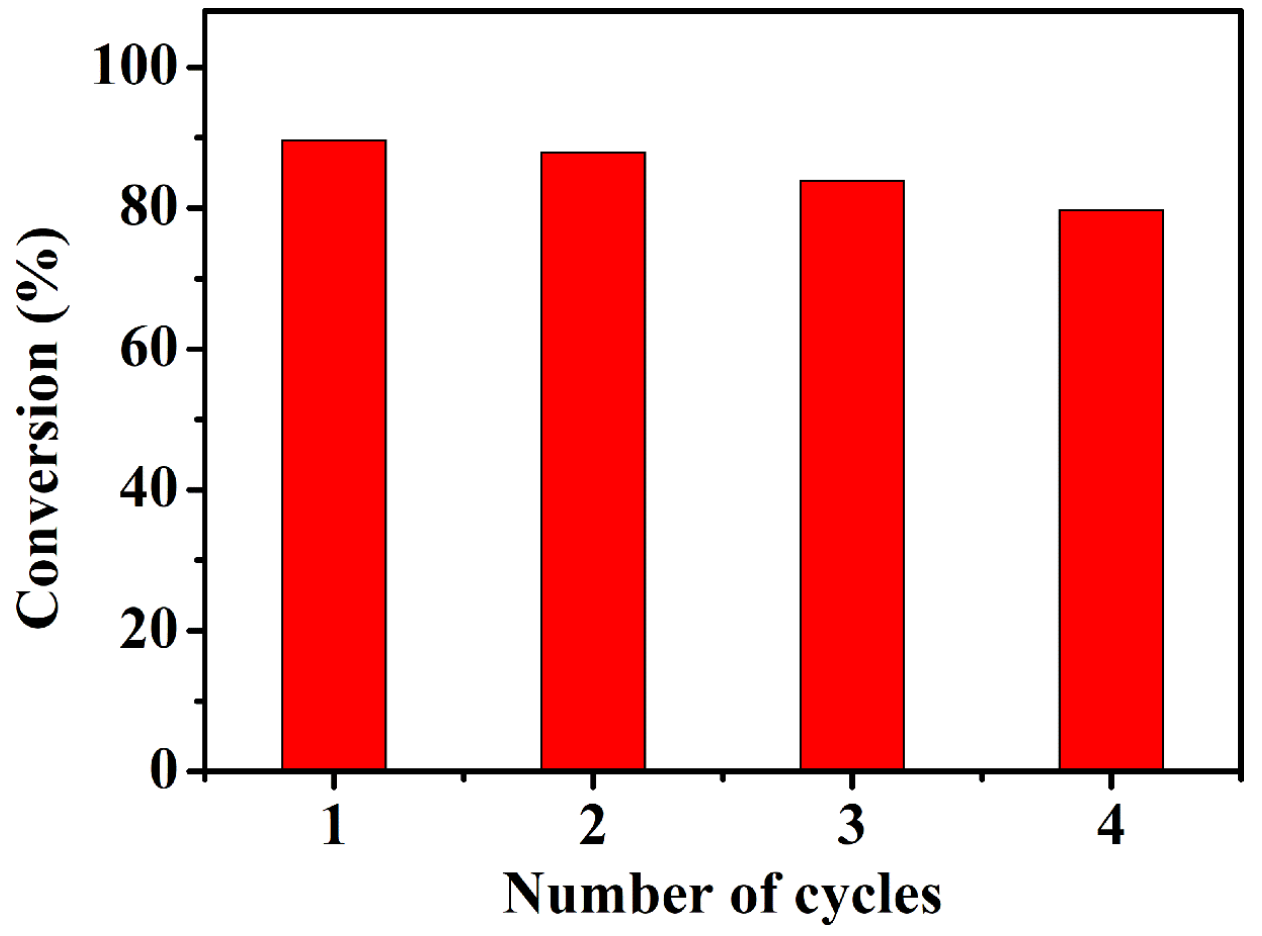

Figure S26. Recyclability of compound 2 as catalyst. 
Section 5. Other Tables

Table S2. Selected bond lengths ( $(\AA)$ and angles $\left(^{\circ}\right)$ for compound 1.

\begin{tabular}{|c|c|c|c|}
\hline $\mathrm{Cu}(1)-\mathrm{O}(1) \# 1$ & $1.961(4)$ & $\mathrm{O}(4) \# 5-\mathrm{Mo}(1)-\mathrm{O}(4) \# 6$ & $88.2(6)$ \\
\hline $\mathrm{Cu}(1)-\mathrm{O}(1) \# 2$ & $1.961(4)$ & $\mathrm{O}(2)-\mathrm{Mo}(1)-\mathrm{O}(3) \# 1$ & $158.5(4)$ \\
\hline $\mathrm{Cu}(1)-\mathrm{O}(1) \# 3$ & $1.961(4)$ & $\mathrm{O}(2)-\mathrm{Mo}(1)-\mathrm{O}(3)$ & $158.5(4)$ \\
\hline $\mathrm{Cu}(1)-\mathrm{O}(1)$ & $1.961(4)$ & $\mathrm{O}(3)-\mathrm{P}(1)-\mathrm{O}(3) \# 7$ & $180.0(15)$ \\
\hline $\mathrm{Cu}(1)-\mathrm{O}(1 \mathrm{~W})$ & $2.195(12)$ & $\mathrm{O}(3)-\mathrm{P}(1)-\mathrm{O}(3) \# 1$ & $70.528(1)$ \\
\hline $\operatorname{Mo}(1)-\mathrm{O}(2)$ & $1.648(10)$ & $\mathrm{O}(3) \# 7-\mathrm{P}(1)-\mathrm{O}(3) \# 1$ & $109.472(8)$ \\
\hline $\operatorname{Mo}(1)-\mathrm{O}(4)$ & $1.907(4)$ & $\mathrm{O}(3)-\mathrm{P}(1)-\mathrm{O}(3) \# 8$ & $109.471(4)$ \\
\hline $\operatorname{Mo}(1)-\mathrm{O}(4) \# 1$ & $1.907(4)$ & $\mathrm{O}(3) \# 7-\mathrm{P}(1)-\mathrm{O}(3) \# 8$ & $70.529(1)$ \\
\hline $\operatorname{Mo}(1)-\mathrm{O}(4) \# 5$ & $1.907(4)$ & $\mathrm{O}(3) \# 1-\mathrm{P}(1)-\mathrm{O}(3) \# 8$ & $70.529(2)$ \\
\hline $\operatorname{Mo}(1)-\mathrm{O}(4) \# 6$ & $1.907(4)$ & $\mathrm{O}(3)-\mathrm{P}(1)-\mathrm{O}(3) \# 9$ & $70.529(7)$ \\
\hline $\operatorname{Mo}(1)-\mathrm{O}(3) \# 1$ & $2.454(12)$ & $\mathrm{O}(3) \# 7-\mathrm{P}(1)-\mathrm{O}(3) \# 9$ & $109.471(1)$ \\
\hline $\mathrm{Mo}(1)-\mathrm{O}(3)$ & $2.454(12)$ & $\mathrm{O}(3) \# 1-\mathrm{P}(1)-\mathrm{O}(3) \# 9$ & $109.471(2)$ \\
\hline $\mathrm{P}(1)-\mathrm{O}(3)$ & $1.56(2)$ & $\mathrm{O}(3) \# 8-\mathrm{P}(1)-\mathrm{O}(3) \# 9$ & $180.0(8)$ \\
\hline $\mathrm{P}(1)-\mathrm{O}(3) \# 7$ & $1.56(2)$ & $\mathrm{O}(3)-\mathrm{P}(1)-\mathrm{O}(3) \# 10$ & 109.5 \\
\hline $\mathrm{P}(1)-\mathrm{O}(3) \# 1$ & $1.56(2)$ & $\mathrm{O}(3) \# 7-\mathrm{P}(1)-\mathrm{O}(3) \# 10$ & $70.529(1)$ \\
\hline $\mathrm{P}(1)-\mathrm{O}(3) \# 8$ & $1.56(2)$ & $\mathrm{O}(3) \# 1-\mathrm{P}(1)-\mathrm{O}(3) \# 10$ & $70.529(2)$ \\
\hline $\mathrm{P}(1)-\mathrm{O}(3) \# 9$ & $1.56(2)$ & $\mathrm{O}(3) \# 8-\mathrm{P}(1)-\mathrm{O}(3) \# 10$ & $109.472(8)$ \\
\hline $\mathrm{P}(1)-\mathrm{O}(3) \# 10$ & $1.56(2)$ & $\mathrm{O}(3) \# 9-\mathrm{P}(1)-\mathrm{O}(3) \# 10$ & $70.528(1)$ \\
\hline $\mathrm{P}(1)-\mathrm{O}(3) \# 11$ & $1.56(2)$ & $\mathrm{O}(3)-\mathrm{P}(1)-\mathrm{O}(3) \# 11$ & $70.529(4)$ \\
\hline $\mathrm{P}(1)-\mathrm{O}(3) \# 12$ & $1.56(2)$ & $\mathrm{O}(3) \# 7-\mathrm{P}(1)-\mathrm{O}(3) \# 11$ & $109.471(4)$ \\
\hline $\mathrm{O}(1) \# 1-\mathrm{Cu}(1)-\mathrm{O}(1) \# 2$ & $169.0(3)$ & $\mathrm{O}(3) \# 1-\mathrm{P}(1)-\mathrm{O}(3) \# 11$ & 109.471(1) \\
\hline $\mathrm{O}(1) \# 1-\mathrm{Cu}(1)-\mathrm{O}(1) \# 3$ & $88.8(3)$ & $\mathrm{O}(3) \# 8-\mathrm{P}(1)-\mathrm{O}(3) \# 11$ & $70.528(1)$ \\
\hline $\mathrm{O}(1) \# 2-\mathrm{Cu}(1)-\mathrm{O}(1) \# 3$ & $90.1(3)$ & $\mathrm{O}(3) \# 9-\mathrm{P}(1)-\mathrm{O}(3) \# 11$ & $109.472(1)$ \\
\hline $\mathrm{O}(1) \# 1-\mathrm{Cu}(1)-\mathrm{O}(1)$ & $90.1(3)$ & $\mathrm{O}(3) \# 10-\mathrm{P}(1)-\mathrm{O}(3) \# 11$ & $180.0(8)$ \\
\hline $\mathrm{O}(1) \# 2-\mathrm{Cu}(1)-\mathrm{O}(1)$ & $88.8(3)$ & $\mathrm{O}(3)-\mathrm{P}(1)-\mathrm{O}(3) \# 12$ & $109.472(1)$ \\
\hline $\mathrm{O}(1) \# 3-\mathrm{Cu}(1)-\mathrm{O}(1)$ & $169.0(3)$ & $\mathrm{O}(3) \# 7-\mathrm{P}(1)-\mathrm{O}(3) \# 12$ & $70.528(2)$ \\
\hline $\mathrm{O}(1) \# 1-\mathrm{Cu}(1)-\mathrm{O}(1 \mathrm{~W})$ & $95.50(13)$ & $\mathrm{O}(3) \# 1-\mathrm{P}(1)-\mathrm{O}(3) \# 12$ & $180.0(8)$ \\
\hline $\mathrm{O}(1) \# 2-\mathrm{Cu}(1)-\mathrm{O}(1 \mathrm{~W})$ & $95.50(13)$ & $\mathrm{O}(3) \# 8-\mathrm{P}(1)-\mathrm{O}(3) \# 12$ & $109.471(4)$ \\
\hline $\mathrm{O}(1) \# 3-\mathrm{Cu}(1)-\mathrm{O}(1 \mathrm{~W})$ & $95.50(13)$ & $\mathrm{O}(3) \# 9-\mathrm{P}(1)-\mathrm{O}(3) \# 12$ & $70.529(3)$ \\
\hline $\mathrm{O}(1)-\mathrm{Cu}(1)-\mathrm{O}(1 \mathrm{~W})$ & $95.50(13)$ & $\mathrm{O}(3) \# 10-\mathrm{P}(1)-\mathrm{O}(3) \# 12$ & 109.471(1) \\
\hline $\mathrm{O}(2)-\mathrm{Mo}(1)-\mathrm{O}(4)$ & $100.8(4)$ & $\mathrm{O}(3) \# 11-\mathrm{P}(1)-\mathrm{O}(3) \# 12$ & $70.529(3)$ \\
\hline $\mathrm{O}(2)-\mathrm{Mo}(1)-\mathrm{O}(4) \# 1$ & $100.8(4)$ & & \\
\hline $\mathrm{O}(4)-\mathrm{Mo}(1)-\mathrm{O}(4) \# 1$ & $88.3(6)$ & & \\
\hline $\mathrm{O}(2)-\mathrm{Mo}(1)-\mathrm{O}(4) \# 5$ & $100.8(4)$ & & \\
\hline $\mathrm{O}(4)-\mathrm{Mo}(1)-\mathrm{O}(4) \# 5$ & $158.3(7)$ & & \\
\hline $\mathrm{O}(4) \# 1-\mathrm{Mo}(1)-\mathrm{O}(4) \# 5$ & $87.7(5)$ & & \\
\hline $\mathrm{O}(2)-\mathrm{Mo}(1)-\mathrm{O}(4) \# 6$ & $100.8(4)$ & & \\
\hline $\mathrm{O}(4)-\mathrm{Mo}(1)-\mathrm{O}(4) \# 6$ & $87.7(5)$ & & \\
\hline $\mathrm{O}(4) \# 1-\mathrm{Mo}(1)-\mathrm{O}(4) \# 6$ & $158.3(7)$ & & \\
\hline
\end{tabular}


Table S3. Selected bond lengths $(\AA)$ and angles $\left({ }^{\circ}\right)$ for compound 2.

\begin{tabular}{|c|c|c|c|}
\hline $\mathrm{Cu}(1)-\mathrm{O}(1)$ & $1.956(4)$ & $\mathrm{O}(2)-\mathrm{Mo}(1)-\mathrm{O}(3)$ & $100.9(3)$ \\
\hline $\mathrm{Cu}(1)-\mathrm{O}(1) \# 1$ & $1.956(4)$ & $\mathrm{O}(3) \# 5-\mathrm{Mo}(1)-\mathrm{O}(3)$ & $158.2(7)$ \\
\hline $\mathrm{Cu}(1)-\mathrm{O}(1) \# 2$ & $1.956(4)$ & $\mathrm{O}(3) \# 6-\mathrm{Mo}(1)-\mathrm{O}(3)$ & $86.9(5)$ \\
\hline $\mathrm{Cu}(1)-\mathrm{O}(1) \# 3$ & $1.956(4)$ & $\mathrm{O}(3) \# 3-\mathrm{Mo}(1)-\mathrm{O}(3)$ & $89.0(5)$ \\
\hline $\mathrm{Cu}(1)-\mathrm{O}(1 \mathrm{~W})$ & $2.179(10)$ & $\mathrm{O}(2)-\mathrm{Mo}(1)-\mathrm{O}(4) \# 3$ & $159.2(4)$ \\
\hline $\mathrm{Mo}(1)-\mathrm{O}(2)$ & $1.617(10)$ & $\mathrm{O}(3) \# 6-\mathrm{Mo}(1)-\mathrm{O}(4) \# 3$ & $94.2(4)$ \\
\hline $\mathrm{Mo}(1)-\mathrm{O}(3) \# 5$ & $1.898(4)$ & $\mathrm{O}(3)-\mathrm{Mo}(1)-\mathrm{O}(4) \# 3$ & $94.2(4)$ \\
\hline $\mathrm{Mo}(1)-\mathrm{O}(3) \# 6$ & $1.898(4)$ & $\mathrm{O}(2)-\mathrm{Mo}(1)-\mathrm{O}(4)$ & $159.2(4)$ \\
\hline $\mathrm{Mo}(1)-\mathrm{O}(3) \# 3$ & $1.898(4)$ & $\mathrm{O}(3) \# 5-\mathrm{Mo}(1)-\mathrm{O}(4)$ & $94.2(4)$ \\
\hline $\mathrm{Mo}(1)-\mathrm{O}(3)$ & $1.898(4)$ & $\mathrm{O}(3) \# 3-\mathrm{Mo}(1)-\mathrm{O}(4)$ & $94.2(4)$ \\
\hline $\mathrm{Mo}(1)-\mathrm{O}(4) \# 3$ & $2.465(11)$ & $\mathrm{O}(2)-\mathrm{V}(1)-\mathrm{O}(3) \# 5$ & $100.9(3)$ \\
\hline $\mathrm{Mo}(1)-\mathrm{O}(4)$ & $2.465(11)$ & $\mathrm{O}(2)-\mathrm{V}(1)-\mathrm{O}(3) \# 6$ & $100.9(3)$ \\
\hline $\mathrm{V}(1)-\mathrm{O}(2)$ & $1.617(10)$ & $\mathrm{O}(3) \# 5-\mathrm{V}(1)-\mathrm{O}(3) \# 6$ & $89.0(5)$ \\
\hline $\mathrm{V}(1)-\mathrm{O}(3) \# 5$ & $1.898(4)$ & $\mathrm{O}(2)-\mathrm{V}(1)-\mathrm{O}(3) \# 3$ & $100.9(3)$ \\
\hline $\mathrm{V}(1)-\mathrm{O}(3) \# 6$ & $1.898(4)$ & $\mathrm{O}(3) \# 5-\mathrm{V}(1)-\mathrm{O}(3) \# 3$ & $86.9(5)$ \\
\hline $\mathrm{V}(1)-\mathrm{O}(3) \# 3$ & $1.898(4)$ & $\mathrm{O}(3) \# 6-\mathrm{V}(1)-\mathrm{O}(3) \# 3$ & $158.2(7)$ \\
\hline $\mathrm{V}(1)-\mathrm{O}(3)$ & $1.898(4)$ & $\mathrm{O}(2)-\mathrm{V}(1)-\mathrm{O}(3)$ & $100.9(3)$ \\
\hline $\mathrm{V}(1)-\mathrm{O}(4) \# 3$ & $2.465(11)$ & $\mathrm{O}(3) \# 5-\mathrm{V}(1)-\mathrm{O}(3)$ & $158.2(7)$ \\
\hline $\mathrm{V}(1)-\mathrm{O}(4)$ & $2.465(11)$ & $\mathrm{O}(3) \# 6-\mathrm{V}(1)-\mathrm{O}(3)$ & $86.9(5)$ \\
\hline $\mathrm{P}(1)-\mathrm{O}(4)$ & $1.519(19)$ & $\mathrm{O}(3) \# 3-\mathrm{V}(1)-\mathrm{O}(3)$ & $89.0(5)$ \\
\hline $\mathrm{P}(1)-\mathrm{O}(4) \# 7$ & $1.520(19)$ & $\mathrm{O}(2)-\mathrm{V}(1)-\mathrm{O}(4) \# 3$ & $159.2(4)$ \\
\hline $\mathrm{P}(1)-\mathrm{O}(4) \# 8$ & $1.520(19)$ & $\mathrm{O}(3) \# 6-\mathrm{V}(1)-\mathrm{O}(4) \# 3$ & $94.2(4)$ \\
\hline $\mathrm{P}(1)-\mathrm{O}(4) \# 9$ & $1.520(19)$ & $\mathrm{O}(3)-\mathrm{V}(1)-\mathrm{O}(4) \# 3$ & $94.2(4)$ \\
\hline $\mathrm{P}(1)-\mathrm{O}(4) \# 10$ & $1.520(19)$ & $\mathrm{O}(2)-\mathrm{V}(1)-\mathrm{O}(4)$ & $159.2(4)$ \\
\hline $\mathrm{P}(1)-\mathrm{O}(4) \# 11$ & $1.520(19)$ & $\mathrm{O}(3) \# 5-\mathrm{V}(1)-\mathrm{O}(4)$ & $94.2(4)$ \\
\hline $\mathrm{P}(1)-\mathrm{O}(4) \# 12$ & $1.520(19)$ & $\mathrm{O}(3) \# 3-\mathrm{V}(1)-\mathrm{O}(4)$ & $94.2(4)$ \\
\hline $\mathrm{P}(1)-\mathrm{O}(4) \# 3$ & $1.520(19)$ & $\mathrm{O}(4)-\mathrm{P}(1)-\mathrm{O}(4) \# 7$ & $70.529(5)$ \\
\hline $\mathrm{O}(1)-\mathrm{Cu}(1)-\mathrm{O}(1) \# 1$ & $89.7(3)$ & $\mathrm{O}(4)-\mathrm{P}(1)-\mathrm{O}(4) \# 8$ & $109.471(6)$ \\
\hline $\mathrm{O}(1)-\mathrm{Cu}(1)-\mathrm{O}(1) \# 2$ & $169.0(3)$ & $\mathrm{O}(4) \# 7-\mathrm{P}(1)-\mathrm{O}(4) \# 8$ & $180(2)$ \\
\hline $\mathrm{O}(1) \# 1-\mathrm{Cu}(1)-\mathrm{O}(1) \# 2$ & $89.3(3)$ & $\mathrm{O}(4)-\mathrm{P}(1)-\mathrm{O}(4) \# 9$ & $180.0(13)$ \\
\hline $\mathrm{O}(1)-\mathrm{Cu}(1)-\mathrm{O}(1) \# 3$ & $89.3(3)$ & $\mathrm{O}(4) \# 7-\mathrm{P}(1)-\mathrm{O}(4) \# 9$ & $109.471(1)$ \\
\hline $\mathrm{O}(1) \# 1-\mathrm{Cu}(1)-\mathrm{O}(1) \# 3$ & $169.0(3)$ & $\mathrm{O}(4) \# 8-\mathrm{P}(1)-\mathrm{O}(4) \# 9$ & 70.5 \\
\hline $\mathrm{O}(1) \# 2-\mathrm{Cu}(1)-\mathrm{O}(1) \# 3$ & $89.7(3)$ & $\mathrm{O}(4)-\mathrm{P}(1)-\mathrm{O}(4) \# 10$ & $70.529(1)$ \\
\hline $\mathrm{O}(1)-\mathrm{Cu}(1)-\mathrm{O}(1 \mathrm{~W})$ & $95.49(13)$ & $\mathrm{O}(4) \# 7-\mathrm{P}(1)-\mathrm{O}(4) \# 10$ & $109.471(1)$ \\
\hline $\mathrm{O}(1) \# 1-\mathrm{Cu}(1)-\mathrm{O}(1 \mathrm{~W})$ & $95.49(13)$ & $\mathrm{O}(4) \# 8-\mathrm{P}(1)-\mathrm{O}(4) \# 10$ & $70.529(1)$ \\
\hline $\mathrm{O}(1) \# 2-\mathrm{Cu}(1)-\mathrm{O}(1 \mathrm{~W})$ & $95.49(13)$ & $\mathrm{O}(4) \# 9-\mathrm{P}(1)-\mathrm{O}(4) \# 10$ & $109.471(1)$ \\
\hline $\mathrm{O}(1) \# 3-\mathrm{Cu}(1)-\mathrm{O}(1 \mathrm{~W})$ & $95.49(13)$ & $\mathrm{O}(4)-\mathrm{P}(1)-\mathrm{O}(4) \# 11$ & $109.471(1)$ \\
\hline $\mathrm{O}(2)-\mathrm{Mo}(1)-\mathrm{O}(3) \# 5$ & $100.9(3)$ & $\mathrm{O}(4) \# 7-\mathrm{P}(1)-\mathrm{O}(4) \# 11$ & $70.529(2)$ \\
\hline $\mathrm{O}(2)-\mathrm{Mo}(1)-\mathrm{O}(3) \# 6$ & $100.9(3)$ & $\mathrm{O}(4) \# 8-\mathrm{P}(1)-\mathrm{O}(4) \# 11$ & $109.471(5)$ \\
\hline $\mathrm{O}(3) \# 5-\mathrm{Mo}(1)-\mathrm{O}(3) \# 6$ & $89.0(5)$ & $\mathrm{O}(4) \# 9-\mathrm{P}(1)-\mathrm{O}(4) \# 11$ & $70.529(1)$ \\
\hline $\mathrm{O}(2)-\mathrm{Mo}(1)-\mathrm{O}(3) \# 3$ & $100.9(3)$ & $\mathrm{O}(4) \# 10-\mathrm{P}(1)-\mathrm{O}(4) \# 11$ & $180.0(7)$ \\
\hline $\mathrm{O}(3) \# 5-\mathrm{Mo}(1)-\mathrm{O}(3) \# 3$ & $86.9(5)$ & $\mathrm{O}(4)-\mathrm{P}(1)-\mathrm{O}(4) \# 12$ & $109.471(2)$ \\
\hline $\mathrm{O}(3) \# 6-\mathrm{Mo}(1)-\mathrm{O}(3) \# 3$ & $158.2(7)$ & $\mathrm{O}(4) \# 7-\mathrm{P}(1)-\mathrm{O}(4) \# 12$ & $70.529(5)$ \\
\hline
\end{tabular}




\begin{tabular}{llll}
\hline $\mathrm{O}(4) \# 8-\mathrm{P}(1)-\mathrm{O}(4) \# 12$ & $109.471(1)$ & $\mathrm{O}(4) \# 8-\mathrm{P}(1)-\mathrm{O}(4) \# 3$ & $70.529(3)$ \\
$\mathrm{O}(4) \# 9-\mathrm{P}(1)-\mathrm{O}(4) \# 12$ & $70.529(5)$ & $\mathrm{O}(4) \# 9-\mathrm{P}(1)-\mathrm{O}(4) \# 3$ & $109.471(6)$ \\
$\mathrm{O}(4) \# 10-\mathrm{P}(1)-\mathrm{O}(4) \# 12$ & $70.529(5)$ & $\mathrm{O}(4) \# 10-\mathrm{P}(1)-\mathrm{O}(4) \# 3$ & $109.471(6)$ \\
$\mathrm{O}(4) \# 11-\mathrm{P}(1)-\mathrm{O}(4) \# 12$ & $109.471(2)$ & $\mathrm{O}(4) \# 11-\mathrm{P}(1)-\mathrm{O}(4) \# 3$ & $70.529(3)$ \\
$\mathrm{O}(4)-\mathrm{P}(1)-\mathrm{O}(4) \# 3$ & $70.529(3)$ & $\mathrm{O}(4) \# 12-\mathrm{P}(1)-\mathrm{O}(4) \# 3$ & $180(2)$ \\
$\mathrm{O}(4) \# 7-\mathrm{P}(1)-\mathrm{O}(4) \# 3$ & $109.471(3)$ & & \\
\hline
\end{tabular}


Table S4. The hydrogen bond lengths ( $\AA$ ) for compounds 1 and 2.

\begin{tabular}{ccc}
\hline & $\mathrm{D}-\mathrm{H} \cdots \mathrm{A}$ & $\mathrm{D} \cdots \mathrm{A}$ \\
\hline $\mathrm{O} 2 \mathrm{~W}-\mathrm{H} \cdots \mathrm{O} 2 \mathrm{WA}$ & $2.81(2)$ \\
$\mathrm{O} 2 \mathrm{~W}-\mathrm{H} \cdots \mathrm{O} 2 \mathrm{WC}$ & $2.81(2)$ \\
$\mathrm{O} 2 \mathrm{WA}-\mathrm{H} \cdots \mathrm{O} 2 \mathrm{WB}$ & $2.81(2)$ \\
$\mathrm{O} 2 \mathrm{WB}-\mathrm{H} \cdots \mathrm{O} 2 \mathrm{WC}$ & $2.81(2)$ \\
$\mathrm{O} 2 \mathrm{~W}-\mathrm{H} \cdots \mathrm{O} 3 \mathrm{~W}$ & $2.65(3)$ \\
$\mathrm{O} 2 \mathrm{WA}-\mathrm{H} \cdots \mathrm{O} 3 \mathrm{~W}$ & $2.65(3)$ \\
$\mathrm{O} 2 \mathrm{WB}-\mathrm{H} \cdots \mathrm{O} 3 \mathrm{~W}$ & $2.65(3)$ \\
$\mathrm{O} 2 \mathrm{WC}-\mathrm{H} \cdots \mathrm{O} 3 \mathrm{~W}$ & $2.65(3)$ \\
\hline $\mathrm{O} 2 \mathrm{Wa}-\mathrm{H} \cdots \mathrm{O} 2 \mathrm{~Wb}$ & $2.74(2)$ \\
$\mathrm{O} 2 \mathrm{Wa}-\mathrm{H} \cdots \mathrm{O} 2 \mathrm{Wd}$ & $2.74(2)$ \\
$\mathrm{O} 2 \mathrm{Wa}-\mathrm{H} \cdots \mathrm{O} 3 \mathrm{We}$ & $2.99(2)$ \\
$\mathrm{O} 2 \mathrm{Wa}-\mathrm{H} \cdots \mathrm{O} 3 \mathrm{Wh}$ & $2.99(2)$ \\
$\mathrm{O} 2 \mathrm{~Wb}-\mathrm{H} \cdots \mathrm{O} 3 \mathrm{We}$ & $2.99(2)$ \\
$\mathrm{O} 2 \mathrm{Wd}-\mathrm{H} \cdots \mathrm{O} 3 \mathrm{Wh}$ & $2.99(2)$ \\
$\mathrm{O} 3 \mathrm{Wa}-\mathrm{H} \cdots \mathrm{O} 3 \mathrm{Wj}$ & $3.53(3)$ \\
$\mathrm{O} 3 \mathrm{Wa}-\mathrm{H} \cdots \mathrm{O} 3 \mathrm{Wh}$ & $3.53(3)$ \\
$\mathrm{O} 3 \mathrm{Wh}-\mathrm{H} \cdots \mathrm{O} 3 \mathrm{Wj}$ & $3.53(3)$ \\
$\mathrm{O} 3 \mathrm{~Wb}-\mathrm{H} \cdots \mathrm{O} 3 \mathrm{Wi}$ & $3.53(3)$ \\
$\mathrm{O} 3 \mathrm{Wi}-\mathrm{H} \cdots \mathrm{O} 3 \mathrm{We}$ & $3.53(3)$ \\
$\mathrm{O} 3 \mathrm{~Wb}-\mathrm{H} \cdots \mathrm{O} 3 \mathrm{We}$ & $3.53(3)$ \\
\hline
\end{tabular}

Symmetry codes: A: -z, y, x; B: -x, y, -z; C: z, y -x; a: 0.5+y, z, 0.5+x; b: 0.5-x, z, 0.5+y; c: 0.5-y, z, 0.5-x; d: 0.5+x, z, 0.5-y; e: 0.5-y, x, 0.5+z; f: 0.5-z, x, 0.5-y; g: $0.5+y, x, 0.5-z ; h: 0.5+z, x, 0.5+y, h: 0.5+z . x, 0.5+y, i: 0.5-z, y, 0.5+x, j: 0.5+x, y$, $0.5+\mathrm{z}$. 
Table S5. The comparison of benzene hydroxylation of the reported POM-based catalysts.

\begin{tabular}{|c|c|c|c|c|}
\hline Catalyst & $\begin{array}{c}\text { Time/ } \\
\mathrm{h} \\
\end{array}$ & $\mathrm{T} /{ }^{\circ} \mathrm{C}$ & $\begin{array}{l}\text { Phenol } \\
\text { yield/\% }\end{array}$ & Ref \\
\hline$\left[\mathrm{Mo}_{2} \mathrm{~V}_{2} \mathrm{O}_{9}(\mathrm{bpy})_{6}\right]\left[\mathrm{PMo}_{11} \mathrm{VO}_{40}\right]$ & 2 & 80 & 25.5 & 1 \\
\hline $\mathrm{PMoV}_{2} / \mathrm{DMA} 16-\mathrm{CMPS}$ & 9 & 65 & 21.9 & 2 \\
\hline$\left[\mathrm{Cu}_{12}(\mathrm{BTC})_{8}\left(\mathrm{H}_{2} \mathrm{O}\right)_{12}\right]\left[\mathrm{H}_{5} \mathrm{PMo}_{10} \mathrm{~V}_{2} \mathrm{O}_{40}\right] @ 49 \mathrm{H}_{2} \mathrm{O}$ & 4 & 65 & 13.4 & $\begin{array}{r}\text { This } \\
\text { work }\end{array}$ \\
\hline [DiBimCN $]_{2} \mathrm{HPMoV}_{2} @ \mathrm{NC}-580$ & 17 & 140 & 10.5 & 3 \\
\hline$\left(\left[\left(\mathrm{CH}_{3}\right)_{4} \mathrm{~N}\right]_{6}\left[\mathrm{PMo}_{9} \mathrm{~V}_{3} \mathrm{O}_{40}\right]\left[\mathrm{Cu}_{2}\left(\mathrm{C}_{6} \mathrm{H}_{3} \mathrm{O}_{6}\right)_{4 / 3}\right]_{6}\right)$ & 1.33 & 80 & 9.93 & 4 \\
\hline POM@MOF-199@SBA-15 & 0.33 & 80 & 6 & 5 \\
\hline
\end{tabular}

Table S6. The comparison of DBT's removal of the reported POM-based catalysts.

\begin{tabular}{|c|c|c|c|}
\hline Catalyst & $\mathrm{T} /{ }^{\circ} \mathrm{C}$ & DBT conversion. $/ \%$ & Ref \\
\hline$[\mathrm{PyPS}]_{3}\left(\mathrm{NH}_{4}\right)_{3} \mathrm{Mo}_{7} \mathrm{O}_{24}$ & 25 & 99 & 6 \\
\hline $\mathrm{H}_{6} \mathrm{PMo}_{9} \mathrm{~V}_{3} \mathrm{O}_{40} @$ rht-MOF-1 & 70 & 96 & 7 \\
\hline$\left[\mathrm{Cu}_{12}(\mathrm{BTC})_{8}\left(\mathrm{H}_{2} \mathrm{O}\right)_{12}\right]\left[\mathrm{H}_{5} \mathrm{PMo}_{10} \mathrm{~V}_{2} \mathrm{O}_{40}\right] @ 49 \mathrm{H}_{2} \mathrm{O}$ & 65 & 89.6 & This work \\
\hline$[\mathrm{PSPy}]_{3} \mathrm{PW}_{12} \mathrm{O}_{40} \cdot 2 \mathrm{H}_{2} \mathrm{O}$ & 30 & 77.1 & 8 \\
\hline $\mathrm{H}_{5} \mathrm{PW}_{10} \mathrm{~V}_{2} \mathrm{O}_{40} / \mathrm{SiO}_{2}$ & 65 & 70 & 9 \\
\hline$\left[\left(\mathrm{C}_{2} \mathrm{~N}_{2} \mathrm{H}_{8}\right)_{4}\left(\mathrm{CH}_{3} \mathrm{O}\right)_{4} \mathrm{~V}^{\mathrm{IV}}{ }_{4} \mathrm{~V}_{4} \mathrm{O}_{16}\right] \cdot 4 \mathrm{CH}_{3} \mathrm{OH}$ & 40 & 67.6 & 10 \\
\hline
\end{tabular}




\section{Reference}

(1) Yang, H.; Wu, Q.; Li, J.; Dai, W.; Zhang, H.-Y.; Lu, D.; Gao, S.; You, W.-S. Direct Synthesis of Phenol from Benzene Catalyzed by Multi-V-POMs Complex. Applied Catalysis A: General. 2013, 457, 21-25.

(2) Wang, H.-F.; Fang, L.-P.; Yang, Y.-F.; Zhang, L.; Wang, Y.-J. $\mathrm{H}_{5} \mathrm{PMo}_{10} \mathrm{~V}_{2} \mathrm{O}_{40}$ Immobilized on Functionalized Chloromethylated Polystyrene by Electrostatic Interactions: A Highly Efficient and Recyclable Heterogeneous Catalyst for Hydroxylation of Benzene. Catal. Sci. Technol. 2016, 6, 8005-8015.

(3) Cai, X.-C.; Wang, Q.; Liu, Y.-Q.; Xie, J.-Y.; Long, Z.-Y.; Zhou, Y.; Wang, J. Hybrid of Polyoxometalate-Based Ionic Salt and N-Doped Carbon toward Reductant-Free Aerobic Hydroxylation of Benzene to Phenol. ACS Sustain. Chem. Eng. 2016, 4, 4986-4996.

(4) Yang, H.; Li, J.; Wang, L.-Y.; Dai, W.; Lv, Y.; Gao, S. Exceptional Activity for Direct Synthesis of Phenol from Benzene over PMoV@MOF with $\mathrm{O}_{2}$. Catal. Commun. 2013, 35, 101-104.

(5) Yang, H.; Li, J.; Zhang, H.-Y.; Lv, Y.; Gao, S. Facile Synthesis of POM@MOF Embedded in SBA-15 as a Steady Catalyst for the Hydroxylation of Benzene. Microporous Mesoporous Mater. 2014, 195, 87-91.

(6) Hao, L. W.; Sun, L. L.; Su, T.; Hao, D. M.; Liao, W. P.; Deng, C. L.; Ren, W. Z.; Zhang, Y. M.; Lu, H. Y. Polyoxometalate-Based Ionic Liquid Catalyst with Unprecedented Activity and Selectivity for Oxidative Desulfurization of Diesel in $\left[\mathrm{Omin}_{\mathrm{B}} \mathrm{BF}_{4}\right.$. Chem. Eng. J. 2019, 358, 419-426.

(7) Wei, P. P.; Yang, Y.; Li, W. Z.; Li, G. M. Keggin-POM@rht-MOF-1 Composite as Heterogeneous Catalysts towards Ultra-Deep Oxidative Fuel Desulfurization. Fuel. 2020, 274,117834 .

(8) Huang, W.; Zhu, W.; Li, H.; Shi, H.; Zhu, G.; Liu, H.; Chen, G. Heteropolyanion-Based Ionic Liquid for Deep Desulfurization of Fuels in Ionic Liquids. Ind. Eng. Chem. Res. 2010, 49, 8998-9003.

(9) Mansourian, S.-H.; Shahhosseini, S.; Maleki, A. Optimization of Oxidative Polymerization-Desulfurization of a Model Fuel Using Polyoxometalate: Effect of Ultrasound Irradiation. J. Ind. Eng. Chem. 2019, 80, 576-589.

(10) Cao, J. P.; Xue, Y. S.; Li, N. F.; Gong, J. J.; Kang, R. K.; Xu, Y. LA Dominant Windmill-Shaped V8 Clusters: A Bifunctional Heterogeneous Catalyst for $\mathrm{CO}_{2}$ Cycloaddition and Oxidation of Sulfides. J. Am. Chem. Soc. 2019, 141, 19487-19497. 